Abstracts from the

\title{
Abstracts from the NIH Office of Research on Women's Health 2017 Annual BIRCWH Meeting - Building Interdisciplinary Research \\ Careers in Women's Health \\ October 25, 2017
}




\title{
2017 Annual Meeting of the Building Interdisciplinary Research Careers in Women's Health Program
}

\author{
Lisa Begg, Dr.P.H, R.N.
}

The Office of Research on Women's Health (ORWH), National Institutes of Health (NIH), is hosting the annual meeting for its signature career development program in October 2017. The program, Building Interdisciplinary Research Careers in Women's Health (BIRCWH), provides NIH funding to academic institutions that have developed scientifically competitive training programs for mentoring junior faculty, known as BIRCWH Scholars, for future research careers.

ORWH developed, and implemented the BIRCWH institutional career development program in 1999 to increase the number of women's health researchers working in a mentored interdisciplinary environment. The junior faculty members supported by the BIRCWH Program have recently completed clinical training or postdoctoral fellowships. They are conducting basic, translational, clinical, or health services research related to women's health research by pairing them with senior investigators who serve as their mentors. These mentors are more senior-level investigators who have developed NIHfunded research programs. The BIRCWH Program is built around three pillars: strong mentoring, interdisciplinary research, and career development. ${ }^{1}$

The highlights of the 2017 annual meeting are provided below, and are followed by the BIRCWH Scholar abstracts that will be presented at the meeting.

The October 2017 meeting will open with a Keynote address from the executive director of the White House National Science and Technology Council, Office of Science and Technology, on future trends in science and technology. This talk will be followed by a panel of the BIRCWH Principal Investigators and the director of the NIH Division of Biomedical Research Workforce discussing the evolving research environment and technologies that allow collaboration and access to information. Because funding opportunities are increasingly competitive, and research funding is becoming increasingly difficult to obtain, the purpose of this session is to learn from history and experience: what are effective strategies to have a long and successful research career in the face of such adversity? This session will examine trends in data from the NIH; provide advice to Scholars; and discuss tools, resources, and strategies for programs, departments, and institutions to promote immediate and long-term research success of BIRCWH Scholars and alumni. Following the panel will be an important talk focusing on strategies to enhance team-based, crossdisciplinary science, including highlighting current and future trends, and practical tools to use. These resources, contained on the National Cancer Institute's Team Science Toolkit, (www .teamsciencetoolkit.cancer.gov), is a "one-stop-shop" for resources to help lead, manage, evaluate, facilitate, or support team science. The key resources available through the Toolkit can be used by investigators, academic institutions, and funders to maximize the success of team science.

Collectively, the speaker talks and panel discussion will provide information to Principal Investigators and Scholars on facing and overcoming challenges in their career, including those related to career development and advancement and ways to exercise research excellence. The morning plenary session will close out with three (3) BIRCWH Scholars giving presentations of their research. The remainder of the day includes a session on mentoring from a number of NIH Institute and Center staff, and the Poster session.

The 2017 meeting abstracts represent many scientific areas that broadly fall within women's health and sex differences research, and include public health topics, basic, clinical, and translational science projects. The full list of the active BIRCWH programs can be found at: https://orwh.od.nih.gov/career/ mentored/bircwh/.

For further information, please contact Dr. Lisa Begg at the NIH: beggl@od.nih.gov

\footnotetext{
${ }^{1}$ Office of Research on Women's Health (2015) Report of the Advisory Committee on Research on Women's Health FY2013 - 2014: Office of Research on Women's Health and NIH Support for Research on Women's Health. Bethesda, MD: US Department of Health and Human Services, Public Health Service, National Institutes of Health. NIH Publication No. 15-7995. Available at http://orwh.od.nih.gov/ about/acrwh/biennial-report/index.asp.
} 


\section{BIRCWH Presentation Abstracts}

\section{O-1: Understanding the Genetic Basis for Female-Biased Autoimmunity: Unusual X-Chromosome Inactivation in Female Lymphocytes}

Camille Syrett, Montserrat C. Anguera (presenting author)

Department of Biomedical Sciences, University of Pennsylvania

Background and Objectives: Females are more prone to develop autoimmune disorders, and the genetic basis for this susceptibility is due to the $\mathrm{X}$-chromosome imbalance between sexes. Systemic lupus erythomatosis (SLE) patients are predominantly female, and lymphocytes from female SLE patients overexpress X-linked immunity-related genes. The mechanisms responsible for the aberrant increased expression of immunityrelated X-linked genes are unknown. Female mammals use Xchromosome Inactivation (XCI) as a mechanism for dosage compensation of $\mathrm{X}$-linked genes. The inactive $\mathrm{X}(\mathrm{Xi})$ chromosome is maintained transcriptionally silent with each cell division by a variety of epigenetic modifications, from the early embryo into adulthood.

Methods and Results: We recently discovered that XCI in female lymphocytes is uniquely regulated, as the $\mathrm{Xi}$ lacks localized Xist RNA and heterochromatin modifications in resting lymphocytes. Upon cell stimulation, these modifications return to the $\mathrm{Xi}$, and lymphocytes are the only somatic cells that utilize a dynamic mechanism for maintaining XCI. We propose that the Xi in lymphocytes is uniquely poised for selective gene reactivation from the $\mathrm{Xi}$. Our data reveal that these unusual features of the $\mathrm{Xi}$ in mature B cells originate during B cell development. We found that lymphocytes from SLE patients exhibit mis-localized epigenetic modifications on the $\mathrm{Xi}$, together with increased expression of certain X-linked genes.

Conclusions: Together, our results indicate that localization of epigenetic modifications to the $\mathrm{Xi}$ is critical for maintaining $\mathrm{XCI}$ in female lymphocytes, and that chromatin changes on the $\mathrm{Xi}$ during $\mathrm{B}$ cell development and the dynamic nature of $\mathrm{XCI}$ maintenance in mature B cells predisposes X-linked immunity genes to reactivation.

\section{O-2: The Financial Burden of Breast Cancer Treatment}

Rachel Adams Greenup (presenting author), ${ }^{1}$ Laura Fish, ${ }^{2}$ Christel Rushing, ${ }^{3}$ Jeffrey Peppercorn, ${ }^{4}$ Terry Hyslop, ${ }^{3}$ Yousuf Zafar, ${ }^{5}$ and E. Shelley Hwang ${ }^{1}$

${ }^{1}$ Department of Surgery and Duke Cancer Institute, ${ }^{2}$ Duke Cancer Control and Population Sciences, ${ }^{3}$ Department of Biostatistics and Bioinformatics, Duke University, and ${ }^{5}$ Department of Medicine, Duke University; ${ }^{4}$ Department of Medicine, Massachusetts General Hospital

Background and Objectives: The burden of cancer treatment costs result in financial toxicity for many patients. Our objective was to understand the impact of cancer treatment costs on women with breast cancer and to determine their preferences for cost transparency.

Methods: Women $\geq 18$ years old with breast cancer were recruited from the Army of Women to complete a 65-item electronically administered survey.

Results: A total of 508 women responded. Mean age was 58.5 years. Mean time from diagnosis was 7.9 years. Stage at diagnosis was stage $0(19 \%)$, stage $1(36 \%)$, stage $2(31 \%)$, stage 3 $(9.5 \%)$, stage $4(1.4 \%)$, and unknown $(3.4 \%)$. Forty-three percent of women were actively receiving treatment. Insurance status included $65 \%$ Private, $27 \%$ Medicare, $2.8 \%$ Medicaid, $2.0 \%$ Military/VA, $0.2 \%$ uninsured, and 3.4\% unknown. Seventynine percent of women reported never discussing treatment costs with their medical team. Costs of treatment were reported as higher than expected by $32 \%$. Sixty-five percent did not consider costs when making treatment decisions; however, $12 \%$ of women reported that costs were "extremely important." Forty-one percent of women agreed or strongly agreed that doctors should consider costs when making recommendations. Almost one-third of women reported somewhat to catastrophic financial burden, which was greater in Medicaid recipients than the privately insured $(14 \%$ vs. $3 \%)$

Conclusions: Many breast cancer patients report a preference for costs information, and many reported significant financial burden related to their treatment. Improving cost transparency for breast cancer patients may impact treatment decisions.

\section{O-3: Beyond Anatomy: Quantifying Blood Flow in the Heart to Better Phenotype Cardiovascular Disease in Women and Men}

Viviany R. Taqueti (presenting author), Leslee J. Shaw, Nancy R. Cook, Venkatesh L. Murthy, Nishant R. Shah, Courtney R. Foster, Jon Hainer, Ron Blankstein, Sharmila Dorbala, and Marcelo F. Di Carli

Noninvasive Cardiovascular Imaging Program, Division of Cardiovascular Medicine, Department of Medicine and Division of Nuclear Medicine and Molecular Imaging, Department of Radiology, Brigham and Women's Hospital, Harvard Medical School

Background and Objectives: Cardiovascular disease (CVD) fatality rates are higher for women as compared with men, yet obstructive coronary artery disease (CAD) is less prevalent in women. Coronary flow reserve (CFR), an integrated measure of large and small vessel CAD and myocardial ischemia, identifies patients at risk for CVD death but is not routinely measured in clinical practice. We sought to investigate the impact of sex, CFR, and angiographic CAD severity on adverse cardiovascular events.

Methods: Consecutive patients $(\mathrm{N}=329,43 \%$ women $)$ referred for invasive coronary angiography after stress testing with myocardial perfusion positron emission tomography (PET) were 
followed for major adverse CVD events. Extent and severity of angiographic $\mathrm{CAD}$ were estimated using the $\mathrm{CAD}$ prognostic index, and CFR quantified using PET.

Results: Although women relative to men had lower burden of angiographic CAD $(\mathrm{P}<0.001)$, they demonstrated greater risk of CVD events, even after adjustment for traditional risk factors, imaging findings, and early revascularization (adjusted HR $2.05,95 \%$ CI $1.05-4.02, \mathrm{P}=0.03$ ). After also adjusting for CFR, the effect of sex on outcomes was no longer significant. When stratified by sex and CFR, only women with severely impaired CFR demonstrated significantly increased adjusted risk of CVD events $(\mathrm{P}<0.0001, \mathrm{P}$ for interaction $=0.04)$.

Conclusions: Excess cardiovascular risk in women was independently associated with impaired CFR, representing a hidden biological risk, and a phenotype less amenable to revascularization. Impaired CFR, particularly absent severely obstructive CAD, may represent a novel target for CVD risk reduction. 


\section{BIRCWH Poster Abstracts}

\section{P-1: Cigarette Craving Differs by Use of Hormonal Contraceptives}

Alicia Allen (presenting author), ${ }^{1}$ Lynn Eberly,${ }^{2}$ Sharon Allen, ${ }^{3}$ Mustafa al' $\mathrm{Absi}^{4}{ }^{4}$ and Dorothy Hatsukami ${ }^{5}$

${ }^{1}$ Department of Family \& Community Medicine, University of Arizona; ${ }^{2}$ Biostatistics, ${ }^{3}$ Department of Family Medicine \& Community Health, ${ }^{4}$ Department of Behavioral Medicine and

${ }^{5}$ Department of Psychiatry, University of Minnesota

Background and Objectives: Craving for cigarettes varies by menstrual phase, likely due to the effect of the sex hormones progesterone and estradiol. Hormonal contraceptives (HC) significantly alter the menstrual cycle variation of progesterone and estradiol, and up to $50 \%$ of premenopausal smokers use HC. However, little is known about how craving for cigarettes may vary by $\mathrm{HC}$ use. We sought to compare craving among premenopausal women smokers who were naturally cycling (NC) and either in the follicular (NC-F, i.e., 1-14 post onset of menses) or luteal phase (NC-L; i.e., 15-28 post onset of menses) or using cyclical HC (HC-C; e.g., oral contraceptives) or long-acting HC (HC-L; e.g., hormonal injection, hormonal intrauterine device).

Methods: This online cross-sectional survey recruited female smokers between the ages of 18 and 35. Survey questions included in Minnesota Nicotine Withdrawal Scale, which uses a four-point scale to measure craving (single item) and withdrawal (average of seven items).

Results: Participants $(\mathrm{n}=506)$ were, on average ( \pm standard deviation), $20.7 \pm 2.7$ years of age and smoked 7.0 \pm 6.5 cigarettes/day. Craving (average \pm standard error) varied significantly by study group, such that NC-F $(n=48)$ had the highest level of craving $(2.6+0.1)$, followed by HC-C $(n=231 ; 2.4+0.01)$, HC-L $(n=124 ; 2.3+0.1)$, and NC-L $(n=103 ; 2.0+0.2 ; \mathrm{P}=0.015)$. Withdrawal did not differ by study group.

Conclusions: Like endogenous sex hormones, hormonal contraceptives may alter cigarette craving. Additional research is needed to replicate this observation within a stronger study design and explore how these observations may influence attempts at smoking cessation.

\section{P-2: Confidence for Disclosure of Intimate Partner Violence Among Abused Women: Reasons Why Identification and Counseling Are Critical to Women's Health}

Carmen Alvarez (presenting author), Nancy Perrin, Amber Clough, James Case, and Nancy Glass

Johns Hopkins School of Nursing

Background and Objectives: Intimate partner violence (IPV) remains a prevalent public health problem that disproportionately affects women and women's health. The purpose of this study was to determine (1) whether survivor confidence in implementing harm reduction strategies, including disclosing
IPV, differed by type of abuse/violence, and (2) whether health status differed by type of abuse/violence.

Methods: Data are from a larger, multisite, multilevel systems intervention to increase universal education about IPV and women's health. Eligibility criteria included having a current or ex-partner in the last 6 months and having experienced some form of abuse. Of 872 eligible women, 747 completed at least a portion of the survey.

Results: Women who had experienced psychological and physical abuse, as well as sexual assault (including rape), reported significantly $(P<.01)$ lower levels of confidence in harm reduction strategies, including recognizing IPV and telling a provider about the IPV. When compared to women who had not experienced any violence, women who experienced any type of IPV were in poorer health $(P<.001)$. Similarly, women who experienced any type of IPV reported being in more pain than those who had not experienced violence $(P=.018)$. Of note, one-third $(33.8 \%)$ of women that screened positive for IPV reported that they had no need for safety strategies as they were in a healthy relationship.

Conclusions: These findings highlight the importance of not only routine screening for IPV, but also educating women on healthy relationships and the relationship between IPV and negative health outcomes.

\section{P-3: Caregiver Strain Among Mothers of Girls with Attention-Deficit/Hyperactivity Disorder (ADHD)}

Dara E. Babinski (presenting author), Daniel A. Waschbusch, and James G. Waxmonsky

\section{Department of Psychiatry, Pennsylvania State} College of Medicine

Background and Objectives: Very little research has examined parenting stress among mothers of girls with ADHD. Emerging research highlights a broad range of social-emotional difficulties in girls, which may uniquely impact maternal caregiver strain. This study examined predictors of maternal caregiver strain, including severity of ADHD, oppositional defiant disorder (ODD), and borderline personality features (BPF) in a sample of girls with ADHD.

Methods: Ratings of caregiver strain were collected from mothers of 55 girls (ages 7-16) with ADHD using the Caregiver Strain Questionnaire. Measures of ADHD, ODD, and BPF were collected using maternal reports on the Disruptive Behavior Disorders Rating Scale and Borderline Personality Features for Children Scale. Linear regression analyses examined the associations between these child variables and caregiver strain, controlling for child age and parental marital status.

Results: Significant bivariate $(P<.05)$ associations emerged between caregiver strain and severity of ADHD ( $\mathrm{r}=.35)$, ODD symptoms $(\mathrm{r}=.68)$, and BPF $(\mathrm{r}=.49)$. In regression analyses, BPF $(\mathrm{b}=0.35, P=.041)$ and $\mathrm{ODD}(\mathrm{b}=0.64, P<.01)$ explained unique variance in maternal caregiver strain (total $R$-square $=0.69$ ), while the contribution of ADHD severity no longer remained significant.

Conclusions: Our findings indicate an association between girls' social-emotional difficulties and maternal caregiver strain, 
which is consistent with previous research on boys with ADHD. Our work also suggests that BPF, a relatively understudied interpersonal domain in ADHD, uniquely contributes to caregiver strain. Future research should address bidirectional influences between maternal caregiver strain and social-emotional functioning in girls with ADHD to better elucidate mechanisms of social-emotional dysfunction.

\section{P-4: Sex Differences in Longitudinal Association Between Posttraumatic Stress and Non-Medical Prescription Opioid Use}

Christal L. Badour

Department of Psychology, University of Kentucky

Background and Objectives: Adults with posttraumatic stress disorder (PTSD) are 1.5 to 1.8 times more likely than those without PTSD to meet criteria for non-medical prescription opioid use (NMPOU) disorder. Compared to men, women are twice as likely to meet criteria for PTSD, and are more likely to be prescribed opioids in higher doses and for longer durations. Women are also more likely to report using prescription opioids to cope with negative affect and physical pain, to endorse prescription opioids as their primary substance of abuse, and to experience greater functional impairment and psychiatric comorbidity when seeking treatment for NMPOU disorder. The severity of PTSD-avoidance symptoms strongly correlate with NMPOU among women, while arousal/reactivity symptoms most strongly correlate with NMPOU among men, suggesting that there may be sex differences in the comorbidity's maintaining mechanisms. The present cohort study will expand upon limited crosssectional research in this area by examining sex differences in the relationship between PTSD symptoms and NMPOU over time.

Methods: Sixty adults (30 female, 30 male) with PTSD and NMPOU during the past month (May) will complete daily measures of PTSD symptoms, and prescription opioid and other substance use for 4 weeks.

Results: Data collection is ongoing. It is expected that PTSDavoidance symptoms will be more strongly linked to both concurrent and later NMPOU among women compared to men.

Conclusions: This research will increase understanding of sex differences in how these conditions are functionally related in participants' over time, with the aim of informing targeted intervention development for co-occurring NMPOU and PTSD.

\section{P-5: New Electronic Health Record Method Uncovers Increase in Burden of Comorbidities in African-Americans Versus Caucasians with Systemic Lupus Erythematosus}

April Barnado (presenting author), ${ }^{1}$ Robert Carroll, ${ }^{2}$ Carolyn Casey, ${ }^{1}$ Joshua C. Denny, ${ }^{1,2}$ and Leslie J. Crofford ${ }^{1}$

${ }^{1}$ Department of Medicine and ${ }^{2}$ Department of Biomedical Informatics, Vanderbilt University Medical Center

Background and Objectives: Among those with systemic lupus erythematosus (SLE), African-Americans have increased renal disease, compared to Caucasians. Differences in other comorbidities have not been well described. The objective was to use a novel electronic health record (EHR) technique to test for differences in comorbidities in African-Americans versus Caucasians with SLE.
Methods: We used an EHR with 2.8 million subjects and decades of follow-up. We performed phenome-wide association studies (PheWAS) that scan across billing codes comparing African-American versus Caucasian SLE cases and AfricanAmerican SLE cases versus matched controls, given known health disparities in African-Americans. Controls were age, sex, and race matched to SLE cases.

Results: We identified 270 African-Americans and 715 Caucasians with SLE and 1,425 matched African-American controls. In the PheWAS adjusting for age and sex, African-American SLE patients had more comorbidities in every organ system, compared to Caucasians. The most striking included hypertension $\mathrm{OR}=4.25$, $P=5.49 \times 10^{-15}$; renal dialysis $\mathrm{OR}=10.90, P=8.75 \times 10^{-14}$; and pneumonia $\mathrm{OR}=3.57, P=2.32 \times 10^{-8}$. In the PheWAS comparing African-American SLE cases versus controls, SLE cases had more renal, cardiovascular, and infectious diseases $\left(\mathrm{P}<4.25 \times 10^{-15}\right)$.

Conclusions: African-Americans with SLE have an increased comorbidity burden compared to Caucasians with SLE and matched controls. This increase in comorbidities in AfricanAmericans with SLE has not been well described and highlights the need for clinicians to monitor these patients for cardiovascular and infectious complications.

\section{P-6: Cardiovascular Health Promotion Among African-Americans by FAITH! (Fostering African-American Improvement in Total Health): Engaging the Community Through Mobile Technology-Assisted Education}

LaPrincess Brewer (presenting author), ${ }^{1}$ Sharonne Hayes, ${ }^{1}$ Carmen Radecki-Breitkopf, ${ }^{2}$ Sarah Jenkins, ${ }^{3}$

Kandace Lackore, ${ }^{3}$ Lisa Cooper, ${ }^{4}$ and Christi Patten ${ }^{5}$

${ }^{1}$ Department of Cardiovascular Medicine, ${ }^{2}$ Department of Health Sciences Research, and ${ }^{3}$ Department of Health Sciences Research, Division of Biomedical Statistics and Informatics, Mayo Clinic; ${ }^{4}$ Johns Hopkins Center to Eliminate Cardiovascular Health Disparities, Johns Hopkins University School of Medicine; ${ }^{5}$ Department of Psychology and Psychiatry, Mayo Clinic

Background and Objectives: Cardiovascular disease (CVD) remains the leading cause of death among African-American (AA) women. Given expanding mobile technology use among AA women, mobile health (mHealth) interventions are promising avenues to promote cardiovascular health $(\mathrm{CVH})$ within this population. We present the design and baseline findings of an mHealth CVH promotion intervention among AAs enrolled in a digital application (app)-based program of the American Heart Association's Life's Simple 7 (LS7) components (smoking, diet, physical activity, body mass index [BMI], blood pressure, total cholesterol, and glucose).

Methods: Members from five predominately AA Minnesota churches collaborated in a community-based participatory research approach to develop a 10-week digital app CVH promotion program. We classified participants according to ideal, intermediate, and poor $\mathrm{CVH}$ criteria based on health factors and behaviors (LS7).

Results: Thirty-five women enrolled; their mean age was 50.2 (SD, 12.8), $43 \%$ had at least a college education, and $37 \%$ reported an annual household income of $<\$ 35,000$. Greater than half reported being "very comfortable" with use of mobile technologies $(51 \%)$, and the majority $(>90 \%)$ reported using some form of mobile technology. The top three barriers to 
leading a healthy lifestyle were lack of self-motivation (49\%), family and caregiving obligations (40\%), and lack of confidence for behavior change (34\%). Greater participants were classified as poor $\mathrm{CVH}$ for the LS7 components, including physical activity $(35 \%)$, blood pressure (43\%), and BMI (69\%).

Conclusions: Use of mHealth interventions appears feasible in this population. Future reports will focus on the effect of this program on promoting ideal $\mathrm{CVH}$.

\section{P-7: Race/Ethnicity-, Age-, and Sex-Specific Heritability of Inflammatory Risk Traits in a Family-Based Cohort}

Enkhmaa Byambaa (presenting author), Anuurad Erdembileg, Wei Zhang, Kyoungmi Kim, and Lars Berglund

\section{University of California, Davis}

Background and Objectives: The role of genetics in modulating markers of inflammation, a contributor to atherosclerosis, is not well understood. We investigated the heritability of five systemic (C-reactive protein [CRP], fibrinogen, serum amyloid A [SAA], haptoglobin, and $\alpha$-acid glycoprotein [AGP]) and three vascular (pentraxin-3 [PTX-3], sICAM-1, and sVCAM-1) inflammatory markers in a family-based cohort.

Methods: A total of 82 Caucasian and African-American parent-offspring quartets were enrolled (age range 6-74 years). Heritability was estimated by measuring the relative contribution of the genetic component of variance responsible for parent-offspring resemblance and compared by race/ethnicity, age, and sex.

Results: In Caucasians, six out of eight inflammatory markers (SAA, fibrinogen, AGP, haptoglobin, sICAM-1, and sVCAM-1) were heritable, while only four markers (CRP, SAA, AGP, and sICAM-1) were heritable in African-Americans. Overall, heritability for systemic markers (CRP, SAA, fibrinogen, and haptoglobin) was higher and heritability for vascular markers (PTX-3, sICAM-1, and SVCAM-1) was lower in AfricanAmericans versus Caucasians. A marked more than threefold higher heritability was noted in African-Americans versus Caucasians for SAA $(P=0.015)$. Fibrinogen heritability was significantly higher in older $(\geq 47$ years) versus younger $(<47$ years) Caucasian families $(P=0.002)$, while sICAM-1 heritability was diminished in older versus younger African-American families. Overall, sex-specific heritability estimates were similar, indicating that neither sex appeared to have more heritability for traits tested.

Conclusions: Our findings indicate moderate to high heritability for inflammatory risk traits, and that the contribution of genetics to the phenotypic variance of a trait differs by age and race/ethnicity in Caucasians and African-Americans.

\section{P-8: Perinatal Hepatitis C Virus Transmission and Pediatric Screening}

Catherine A. Chappell (presenting author), ${ }^{1}$ Sharon L. Hillier, ${ }^{1}$ David Crowe, ${ }^{2}$ and Elizabeth E. Krans ${ }^{1}$

${ }^{1}$ Department of Obstetrics, Gynecology and Reproductive Sciences, Magee-Womens Research Institute, University of Pittsburgh, and ${ }^{2}$ Magee-Womens Research Institute

Background and Objectives: Chronic hepatitis $\mathrm{C}$ virus (HCV) in pregnant women is increasing and can result in peri- natal transmission. Our objectives were to determine the rate and type of pediatric $\mathrm{HCV}$ testing for children with $\mathrm{HCV}$ exposure during pregnancy and the rate of perinatal $\mathrm{HCV}$ transmission.

Methods: We identified a cohort of pregnant women who delivered at our institution between January 1, 2006 and December 31, 2014. Maternal HCV infection was defined by ICD-9 coding and all laboratory data were extracted from the linked infant electronic medical records (EMR).

Results: Among 88,317 pregnant women, 1,043 (1.2\%) had $\mathrm{HCV}$ infection with 1,035 linked infant EMR. Among HCVexposed infants, $381(37 \%)$ had laboratory tests available in the EMR after 1 year of life. Among these infants, only $115(30 \%)$ were tested for HCV. Eight other infants were tested but did not have any other laboratory tests in the system after 1 year of age. Of 123 tests used to diagnose HCV, 90 (73\%) were antibody tests. Nine tests were uninterpretable due to an antibody test being drawn too early $(N=5)$ or unavailable results $(N=4)$. Evidence of perinatal transmission, defined as positive antibody after 18 months of life or HCV viremia, occurred in $9(7.3 \%)$ exposed infants, with $7(6.1 \%)$ having chronic HCV infection.

Conclusions: In this retrospective cohort of pregnant women with $\mathrm{HCV}$, less than one-third of exposed infants receiving care were screened for HCV. Further studies should consider interventions to increase HCV screening by pediatric primary care providers.

\section{P-9: Sex Differences in Imaging Biomarkers of First Carpometacarpal Osteoarthritis}

Abhijit J. Chaudhari (presenting author), ${ }^{1}$ Brent H. Foster, ${ }^{2}$ Robert M. Szabo, ${ }^{3}$ Christopher O. Bayne, ${ }^{1}$ Nancy E. Lane, ${ }^{4}$ and Robert D. Boutin ${ }^{1}$

${ }^{1}$ Department of Radiology, ${ }^{2}$ Department of Biomedical Engineering, ${ }^{3}$ Department of Orthopedic Surgery, and ${ }^{4}$ Department of Medicine, University of California, Davis

Background and Objectives: First carpometacarpal (CMC) joint osteoarthritis (OA) is more prevalent in women than in men. We hypothesized that imaging biomarkers associated with structure and composition of tissues of the first CMC joint would differ by sex. Knowledge of these differences will assist in understanding first CMC OA pathogenesis for each sex and inform strategies for its treatment. The objectives are to pilot a created MRI protocol for evaluating the tissues of the first CMC joint, and to develop computational tools for characterizing tissue morphometric and compositional variation for each sex.

Methods: We developed methods to delineate the first CMC joint tissues from MRI and determine any differences in their characteristics by sex. We acquired and evaluated MRIs of the first $\mathrm{CMC}$ joint tissues for 12 women and 12 age-matched men without first CMC OA, and 7 women with OA.

Results: Compared to men without OA, women without OA had an overall lower cartilage volume $\left(116.4 \pm 17.1 \mathrm{~mm}^{3}\right.$ vs. $131.2 \pm 20.9 \mathrm{~mm}^{3}$ in men), while the volumetric subluxation ratio of the first metacarpal bone was higher in women without OA $(0.28 \pm 0.05$ vs. $0.20 \pm 0.07$ in men). In women with OA, the cartilage volume was lower $\left(52.3 \pm 20.0 \mathrm{~mm}^{3}\right)$ and volumetric subluxation ratio higher $(0.36 \pm 0.05)$, compared to both women and men without OA.

Conclusions: In this pilot study, the proposed methods appeared to provide a high sensitivity for detecting specific morphometric and compositional differences by sex for the tissues of the first CMC joint. We will continue to assess the role of these sex differences for sex-specific OA risk assessment and treatment. 


\section{P-10: The Relationship Between Diabetes, Hyperglycemia, and Mortality Among Hispanic and Non-Hispanic White Breast Cancer Survivors}

Avonne E. Connor (presenting author), ${ }^{1}$ Kala Visvanathan, ${ }^{1}$ Stephanie D. Boone, ${ }^{2}$ Kathy B. Baumgartner, ${ }^{2}$ and Richard N. Baumgartner ${ }^{2}$

${ }^{1}$ Department of Epidemiology, Johns Hopkins Bloomberg School of Public Health; and ${ }^{2}$ Department of Epidemiology and Population Health, University of Louisville

Background and Objectives: Research suggests that breast cancer (BC) patients with Type 2 diabetes have an increased risk of mortality, compared to BC survivors without diabetes. Uncontrolled diabetes can result in hyperglycemia. Few diabetesmortality studies have included Hispanic women, in whom diabetes is prevalent. We examined the associations between diabetes, plasma fructosamine-a biomarker of hyperglycemia and glycemic control-and mortality among Hispanic and nonHispanic white (NHW) women diagnosed with invasive BC from the New Mexico Health, Eating, Activity, and Lifestyle cohort.

Methods: A total of 399 BC survivors (96 Hispanic, 303 NHW) contributed baseline data and plasma samples. Hazard ratios (HR) and 95\% confidence intervals (CI) were calculated using multivariable Cox proportional hazards regression models. The percentage change in the HRs to examine mediation due to fructosamine was computed: $\left[\mathrm{HR}_{\text {without fructosamine }}-\right.$ $\left.\left.\left.\mathrm{HR}_{\text {with fructosamine }}\right) / \mathrm{HR}_{\text {without fructosamine }}-1.0\right)\right] \times 100$.

Results: The median follow-up time from baseline interview to death was 11 years. Diabetes was associated with increased mortality overall (HR, 2.03; 95\% CI 1.16-3.53), with stronger effects among Hispanics (HR, 3.16; 95\% CI 1.08-9.23) compared to NHWs (HR, 1.56; 95\% CI 0.77-3.19). Fructosamine was positively associated with mortality overall $(P=0.02)$ and among Hispanics $(P=0.003)$ but not among NHWs $(P=0.64)$. Fructosamine explained $23 \%$ of the diabetes-mortality association overall $\left(\mathrm{HR}_{\mathrm{with}}\right.$ fructosamine $\left.=1.57\right), 38 \%$ of the association among Hispanics $\left(\mathrm{HR}_{\mathrm{with}}\right.$ fructosamine $\left.=1.97\right)$, and only $7 \%$ among NHWs $\left(\mathrm{HR}_{\text {with fructosamine }}=1.45\right)$.

Conclusions: Our results suggest that poor glycemic control might explain a large extent of the relationship between diabetes and mortality after BC, particularly among Hispanics.

\section{P-11: Get Fit and Quit: A Pilot Tobacco Treatment Intervention for Women in a Residential Substance Use Disorder Treatment Facility}

Amanda Fallin-Bennett

College of Nursing, University of Kentucky

Background and Objectives: Smoking is the leading cause of preventable death and disease nationwide. Smoking rates have dramatically decreased among the general population but remain high among vulnerable women, including those with substance use disorders (SUDs). Based on formative qualitative research with women with SUDs, we are piloting Get Fit and Quit (GFAQ), a holistic tobacco treatment intervention. GFAQ's group-care approach provides a unique opportunity for women with SUDs to have customized tobacco treatment in combination with social support and group physical activity for stress reduction. Study aims are to (1) test feasibility and acceptability of the GFAQ program and (2) determine the impact of the GFAQ program on smoking behaviors.

Methods: GFAQ is currently in progress. It is a 10-session program held at a local community Young Men's Christian Association that will occur over a 6-month period. Each session includes (1) evidence-based tobacco treatment groups facilitated by a nurse specializing in tobacco treatment and (2) group physical activity for stress management. Outcomes will be measured at baseline, 4 weeks, 3 months, and 6 months, using surveys and biochemical validation of smoking. Post-program focus groups will evaluate GFAQ acceptability.

Results: Sixteen participants initially enrolled in GFAQ; 14 participants remained engaged at week 5 . We anticipate that GFAQ will be positively viewed, attendance throughout the program will be high, and there will be a reduction in tobacco use.

Conclusions: We anticipate these findings will lead to the development of an innovative tobacco treatment program for dissemination to women's residential SUD treatment programs.

\section{P-12: Metabolomic Pathways in Cardiometabolic Complications of Pregnancy}

Erin P. Ferranti (presenting author), ${ }^{1}$ Jennifer K. Frediani, ${ }^{2}$ Elizabeth J. Corwin, ${ }^{1}$ and Anne L. Dunlop ${ }^{1}$

${ }^{1}$ Nell Hodgson Woodruff School of Nursing and ${ }^{2}$ Department of Pediatrics, School of Medicine, Emory University

Background and Objectives: Specific metabolites have been associated with cardiometabolic complications of pregnancy; however, studies in African-American (AA) women are lacking. The purpose of this study was to investigate metabolomics pathways in early gestation that associate with a later diagnosis of a cardiometabolic complication of pregnancy, including gestational diabetes, gestational hypertension, and/or preeclampsia, in AA women.

Methods: We conducted a retrospective analysis of pregnant AA women 18-40 years of age who had cardiometabolic complications of pregnancy $(n=26)$ and who had uncomplicated, full-term pregnancies $(n=98)$. Participants had serum samples collected between 8-14 weeks gestation and had medical chart abstraction following pregnancy to identify cardiometabolic complications. High-resolution liquid chromatography mass spectrometry (LC-MS) was used to measure serum metabolites.

Results: Significant differences in several metabolic pathways were identified for women with and without cardiometabolic complications of pregnancy, including pathways related to the metabolism of tryptophan, a precursor of serotonin known to be involved in gut function, and pathways related to the metabolism of ascorbate and aldarate, antioxidants that participate in important redox reactions. In the tryptophan pathway, four metabolites were downregulated in the cardiometabolic complications group and 10 were upregulated. In the anti-oxidant ascorbate and aldarate metabolic pathways, three metabolites were downregulated and one upregulated for the cardiometabolic complications group.

Conclusions: Findings suggest a contribution for specific metabolic pathways in the pathogenesis of cardiometabolic complications of pregnancy. Larger scale studies investigating the value of metabolites in these pathways in predicting cardiometabolic complications of pregnancy early in gestation are warranted. 


\section{P-13: Nociceptive Neurons Contribute to Humoral Immune Responses}

Simmie Foster (presenting author), ${ }^{1,2,3}$ Francesco Borriello, ${ }^{4}$ Hannah Merrison, ${ }^{5}$ David Dowling, ${ }^{4}$ Sebastien Talbot, ${ }^{5}$ Corey Seehus, ${ }^{5}$ Ofer Levy, ${ }^{6}$ and Clifford Woolf ${ }^{5,7}$

${ }^{1}$ Department of Psychiatry, Harvard Medical School; ${ }^{2}$ Depression Clinical Research Program, Massachusetts General Hospital; ${ }^{3}$ Harvard BIRCWH Scholar, Brigham and Women's Hospital; ${ }^{4}$ Department of Pediatrics, ${ }^{5}$ FM Kirby Neurobiology Center, and ${ }^{6}$ Department of Medicine, Boston Children's Hospital; ${ }^{7}$ Department of Neurobiology, Harvard Medical School

Background and Objectives: Vaccines against diseases, such as polio and small pox, have been essential for protecting global health, yet the formula behind an effective vaccine for several illnesses remains elusive. All vaccines require an adjuvant, a substance that activates immune cells to recognize an antigen as dangerous. Intriguingly, adjuvants are painful, and nociceptors and immune cells express similar arrays of receptors for adjuvants. These observations led us to hypothesize that the physical pain that vaccine adjuvants induce contributes to the humoral immune response. (1) Do nociceptors respond directly to vaccines, or are immune cells required? (2) Does silencing nociceptors alter antibody responses?

Methods: To answer these questions, we first stimulated nociceptors isolated from mouse dorsal root ganglions with vaccine formulations used in patients and performed calcium imaging. In addition, we immunized mice with intact or ablated nociceptors with a clinically relevant antigen in combination with different adjuvants.

Results: We have found that several clinically relevant vaccines directly activate calcium flux in cultured dorsal root ganglia. Furthermore, nociceptor-ablated mice have an altered humoral response after immunization.

Conclusions: Our results suggest vaccines directly activate nociceptors, thus causing the pain associated with vaccination, and this activation may be important for developing certain types of antibody responses. In addition, previous reports suggest that there is significant sex difference in vaccine efficacy in humans, and also with pain. Our future studies will address whether these observations are related to our findings.

\section{P-14: Uptake of Prenatal Tdap Vaccination in Low- Income and Multiparous Women}

Erika Fuchs (presenting author), ${ }^{1}$ Jonathan Starkey, ${ }^{2}$ and Abbey B. Berenson ${ }^{1}$

${ }^{1}$ Center for Interdisciplinary Research in Women's Health, Department of Obstetrics and Gynecology, and ${ }^{2}$ Institute for Translational Sciences, The University of Texas Medical Branch

Background and Objectives: Tetanus, diphtheria, and acellular pertussis (Tdap) vaccination is recommended during each pregnancy to protect infants from pertussis. Coverage estimates have varied widely and focused on privately insured patients. This study's objective is to examine predictors of prenatal Tdap vaccination in low-income women.

Methods: A retrospective cohort study was conducted using electronic medical records from 15,488 deliveries to 14,942 mothers occurring from April 2, 2014, to December 3, 2016, at a safety net hospital in Texas. Chi-squared tests and t-tests examined differences in Tdap uptake by age, year, race/ethnicity, Medicaid use, and Adequacy of Prenatal Care Utilization index score while unadjusted and adjusted logistic regression estimated odds ratios (OR). Analyses were conducted using Stata/ SE Version 14.2 with significance assessed at $P \leq 0.05$.

Results: Prenatal Tdap uptake increased over time from $52.1 \%$ in 2014 to $71.6 \%$ in 2016 . At mothers' first births in this time period $(n=14,942)$, those who received Tdap were older (27.6 vs. 27.1 years), more likely to have Medicaid-funded births (67.0\% vs. $64.1 \%)$, and less likely to have adequate prenatal care (72.4\% vs. $62.3 \%)$. Tdap uptake was highest among those who identified as Hispanic white $(73.3 \%)$ and lowest among nonHispanic whites $(55.1 \%)$. Among those who delivered more than once in this time period $(n=541)$, mothers who received Tdap in their first pregnancy were more likely to receive it in their second pregnancy $(79.1 \%$ vs. $65.1 \%$, adjusted OR $1.98,95 \%$ confidence interval: 1.29-3.03).

Conclusions: Traditionally at-risk populations may be more likely to receive prenatal Tdap vaccination.

\section{P-15: Thinking Across Generations: Unique Contributions of Maternal Early Life and Prenatal Stress to Infant Autonomic Reactivity}

Sarah A. O. Gray (presenting author), ${ }^{1}$ Christopher W. Jones, ${ }^{2}$ Katherine P. Theall, ${ }^{3}$ and Stacy S. Drury ${ }^{4}$

${ }^{1}$ Department of Psychology, School of Science \& Engineering, ${ }^{2}$ Neuroscience Program, School of Science \& Engineering, ${ }^{3}$ Department of Global Community Health \& Behavioral Sciences, School of Public Health and Tropical Medicine, and ${ }^{4}$ Department of Psychiatry, School of Medicine, The Brain Institute, Tulane University

Background and Objectives: Respiratory Sinus Arrhythmia (RSA) is a parasympathetic-mediated biomarker of regulation linked to physical and mental health outcomes across the lifespan. Intergenerational impacts of mothers' exposure to stress have been demonstrated in other physiological systems, but evidence for biological embedding of maternal early life stress on infant parasympathetic activity is lacking. We examine independent effects of maternal early life and prenatal stress on infant RSA, seeking to broaden the understanding of the fetal origins of physical and mental health vulnerability.

Methods: Mothers $(n=167)$ reported on experiences of early life and prenatal stress during pregnancy. RSA was recorded continuously in 4-month-old infants during a dyadic stressor, the Still Face Paradigm.

Results: Independent contributions of mothers' early life and prenatal stress to infants' parasympathetic functioning were observed. High maternal early life stress was associated with lower RSA levels, whereas prenatal stress was associated with failure to recover following the stressor. Sex but not race differences were observed. Prenatal stress was associated with higher parasympathetic nervous system activity among boys but lower activity among girls.

Conclusions: These findings suggest that infants' emergent parasympathetic reactivity is impacted by their mothers' lifecourse experiences of stress, consistent with prenatal programming and developmental origins theories, with early life stress predicting a lower "set point" and a prenatal stress dampening recovery from stress. For prenatal but not early life stress, patterns vary between boys and girls. These findings underscore the 
importance of early intervention, including during pregnancy, for mothers with high stress to improve maternal and child health outcomes.

\section{P-16: Trends in Breast Cancer Survival Among Young Women 18-39 Years: Effectiveness of Cancer Treatment, Surveillance, Epidemiology, and End Results (SEER) 1973-2014}

Fangjian Guo (presenting author), ${ }^{1,2}$ Yong-Fang Kuo, ${ }^{2,3,4}$ Ya Chen Tina Shih, ${ }^{5}$ and Abbey B. Berenson ${ }^{1}$

${ }^{1}$ Department of Obstetrics \& Gynecology, ${ }^{2}$ Center for Interdisciplinary Research in Women's Health, ${ }^{3}$ Office of Biostatistics, Department of Preventive Medicine and Community Health, and ${ }^{4}$ Institute for Translational Science, The University of Texas Medical Branch; and Department of Health Services Research, The University of Texas MD Anderson Cancer Center

Background and Objectives: Scientific advance in breast cancer treatments has greatly improved survival in patients. However, quantifying effectiveness of treatment advances on cancer survival faces the challenge that screening mammography also contributes to improved survival. This study quantifies the effectiveness of treatment on cancer survival among young female breast cancer patients whose survival was unlikely impacted by screening.

Methods: The study cohort consisted of female breast cancer patients diagnosed between age 18 and 39 from SEER Program 9 registry areas, 1973-2014. Followup was through December 2014. Cox proportional hazard models were fitted to compare differences in 5-year survival probability across time by stage at diagnosis, controlling for age at diagnosis and race.

Results: Among 40,616 breast cancer patients 18-39 years old, observed 5-year breast cancer-specific survival increased significantly from $73.8 \%$ in $1973-1978$ to $88.7 \%$ in $2009-2014$ (adjusted hazard ratio [AHR] for dying from any cause of 2009 2014 vs. 1973-1978 0.38, 95\% confidence interval [CI] 0.34 $0.43)$. The increase in cancer-specific survival reached plateau in 2004, but in metastatic breast cancer it continued to increase after 2004, from $43.3 \%$ in $2004-2008$ to $55.0 \%$ in $2009-2014$ (AHR for 2009-2014 vs. 2004-2008 0.75, 95\% CI 0.61-0.92). Similar patterns were also observed for 5-year all-cause survival.

Conclusions: There were great improvements in the effectiveness of breast cancer treatment on overall survival and cancer-specific survival during 1973-2014. However, the improvements have plateaued since 2004, except that the effectiveness of treatment for metastatic breast cancer continues to improve throughout 1973-2014.

\section{P-17: “Girls in My Ethnic Group Are Angry All of the Time:" The Effects of Gendered Racial Stereotypes of Anger on Girls' Disruptive Behavior}

Colleen A. Halliday-Boykins (presenting author), ${ }^{1}$ Robyn Do, ${ }^{2}$ and Carla Kmett Danielson ${ }^{1}$

${ }^{1}$ Department of Psychiatry and Behavioral Sciences and

${ }^{2}$ Department of Medicine, Medical University of South Carolina

Background and Objectives: One of the most pernicious aspects of racial discrimination is that members of racial mi- nority groups are themselves influenced by negative stereotypes about their groups. For example, one stereotype adopted by some African-American girls and women is that African-American women are loud, hostile, argumentative, and aggressive. Girls' endorsement of such stereotypes can serve as subjective norms about acceptable conduct for girls in their racial group that influence behavior. Thus, identifying links between adoption of gendered racial stereotypes and disruptive behavior might improve understanding of girls' problem behaviors and racial disparities in these outcomes. The purpose of this investigation was to examine the effects of adoption of gendered racial stereotypes of anger on girls' disruptive behavior and examine whether anger expression mediated these associations.

Methods: Participants were 54 middle school girls $(25.0 \%$ African-American, 50.0\% Caucasian). They completed measures of adoption of gendered racial stereotypes, reactive and instrumental aggression, teacher defiance, externalizing symptoms, and anger expression. Data were analyzed using multiple linear regression and the Sobel test of mediation.

Results: Adoption of gendered racial stereotypes was positively related to reactive aggression, teacher defiance, and externalizing symptoms, and these effects were mediated by anger expression. Adoption of gendered racial stereotypes was unrelated to instrumental aggression.

Conclusions: Intervention efforts to improve disruptive behavior in girls may be undermined if gendered racial stereotypes of anger are not addressed. Future research is needed to improve understanding of the role of gendered racial stereotypes in girls' mental health and behavioral outcomes.

\section{P-18: The PPAR $\gamma$ Agonist, Pioglitazone, Confers Sex-Specific Protection from Influenza Virus Infection in Mice}

E. Scott Halstead (presenting author), ${ }^{1,2}$ Sahil Amin, ${ }^{2}$ Todd M. Umstead, ${ }^{1,2}$ Sanmei Hu, ${ }^{1,2}$ and Zissis C. Chroneos ${ }^{1,2}$

${ }^{1}$ Department of Pediatrics and ${ }^{2}$ Pulmonary Immunology and Physiology Laboratory, Pennsylvania State University College of Medicine

Background and Objectives: Female sex was a mortality risk factor during the $2009 \mathrm{H} 1 \mathrm{~N} 1$ influenza A virus (IAV) pandemic. Sex differences in IAV pathogenicity also have been observed in mice, but the underlying mechanism is unclear. We hypothesized that mice exhibit sex-specific differences in antiinflammatory signaling through peroxisome proliferator-activated receptor gamma (PPAR $\gamma$ ). Our objective was to determine whether administering the PPAR $\gamma$ agonist, pioglitazone, confers sexspecific protection IAV infection.

Methods: C57BL/6 wild-type mice were infected intranasally with the PR8 influenza strain. Mice were administered pioglitazone via oral gavage and susceptibility was ascertained by weight loss/recovery and mortality. We performed macrophage transcriptome analysis by fluorescence-activated cell sorting and RNA-sequencing. Ingenuity Pathway Analysis (IPA) was used to generate activation scores for macrophage signaling pathways.

Results: The administration of pioglitazone prior to IAV infection protected both male $(P<0.02)$ and female $(P<0.05)$ mice. Pioglitazone administered after IAV infection protected only male mice $(P<0.02)$. RNA-seq analysis demonstrated decreased expression of PPAR $\gamma$ in female alveolar macrophages $(P=0.03)$. IPA analysis identified that pioglitazone treatment of male mice inhibited multiple inflammatory pathways, including 
STAT1 and the TLR3/4 pathways in alveolar macrophageseffects not seen in female alveolar macrophages.

Conclusions: Pioglitazone confers protection from IAV infection, likely due to its direct effects on alveolar macrophages, which express higher levels of PPAR $\gamma$ as compared to inflammatory macrophages. The therapeutic effect of pioglitazone may be limited in females due to lower macrophage PPAR $\gamma$ expression, though strategies to increase expression may be of benefit and will be a focus of future research.

\section{P-19: Redefining the Bladder Cancer Phenotype Using Sex-Specific Patterns of Familial Risk}

Heidi A. Hanson (presenting author), ${ }^{1}$ Christopher Martin, ${ }^{2}$ Claire Leiser, ${ }^{3}$ Sumati Gupta, ${ }^{4}$ William Lowrance, ${ }^{4}$ Wendy Kohlmann, ${ }^{5}$ Brock O'Neill, ${ }^{2}$ Ken R. Smith, ${ }^{6}$ Orly Alter, ${ }^{7}$ Samantha Greenburg, ${ }^{5}$ and Nicola J. Camp ${ }^{8}$

${ }^{1}$ Department of Surgery, ${ }^{2}$ Division of Urology, Department of Surgery, ${ }^{3}$ Population Sciences, ${ }^{4}$ Division of Oncology, ${ }^{5}$ Population Health Sciences, ${ }^{6}$ Department of Family and Consumer Studies and Population Sciences, Huntsman Cancer Institute; ${ }^{7}$ Department of Bioengineering, and ${ }^{8}$ Internal Medicine, The University of Utah

Background and Objectives: There are large sex-disparities in bladder cancer (BCa). Males have higher incidence, but females have lower rates of survival. Identifying sex-specific risk across a spectrum of related tumors is a fundamental step in elucidating familial and environmental mechanisms predisposing women to $\mathrm{BCa}$ incidence and mortality. We characterize the sex-specific familial clustering of $\mathrm{BCa}$ and related tumors. We investigate sexspecific risk using classic methods with spouses as a proxy for environmental exposures. Next, we attempt to identify new phenotypic classifications utilizing sex-specific patterns of cancer clustering.

Methods: A population-based, case-control study of Utah residents diagnosed with BCa between 1966 and 2014 was used to assess the risk of cancer in family members $(n=4,647)$. Two approaches were used: Cox regression stratified by sex and relation type and k-medoids clustering to identify new familial phenotypes.

Results: First and second-degree relatives of $\mathrm{BCa}$ patients were at increased risk for bladder, lung, kidney, and pancreatic cancer, with no sex-specific risk differences. Significant sex differences in spouses of BCa patients were found. Spouses of female $\mathrm{BCa}$ patients were at increased risk of urothelial $(\mathrm{HR}=1.58)$, kidney $(\mathrm{HR}=1.98)$, and stomach $(\mathrm{HR}=3.32)$. No difference in risk was found for spouses of male $\mathrm{BCa}$ patients. Twelve distinct phenotypes of familial risk were identified with some variability in the sex-difference in BCa risk and mortality between phenotypes.

Conclusions: Considering sex-differences in risk lends evidence to environmental and familial risk factors unique to females and results in more homogenous definitions of bladder cancer phenotypes.

\section{P-20: Which Measures of Pregnancy Ambivalence Are Most Strongly Associated with Contraceptive Non-Use?}

Jenny Higgins (presenting author), ${ }^{1}$ Ronna Popkin, ${ }^{2}$ Emma Carpenter, ${ }^{3}$ Jenny Francis, ${ }^{4}$ and Warren Miller ${ }^{5}$

${ }^{1}$ Department of Gender and Women's Studies, University of Wisconsin; ${ }^{2}$ Department of Sociomedical Sciences and
${ }^{4}$ Department of Pediatrics, Columbia University; ${ }^{3}$ School of Social Work, University of Wisconsin; Columbia University; ${ }^{5}$ Transnational Family Research Institute

Background and Objectives: Research shows associations between pregnancy ambivalence and contraceptive practices. However, pregnancy ambivalence involves many domains, and definitions vary, making study results difficult to interpret. We compared four definitions of pregnancy ambivalence within the same sample and measured their respective associations with contraceptive non-use at last sexual intercourse.

Methods: Using the Fog Zone study, a nationally representative data set of 18-29-year-olds in current sexual relationships $(\mathrm{N}=382$ men, 431 women), we created four measures of pregnancy ambivalence consistent with prior research. We then analyzed the socio-demographic factors and contraceptive non-use patterns most strongly associated with each measure.

Results: The proportion of young adults who qualified as ambivalent about pregnancy ranged from $18-43 \%$ across the measures. With three of the four measures, those classified as ambivalent were significantly less likely to have used contraception in the last month (ORs ranged from $0.30-0.54, \mathrm{P}<.05$ ), even when controlling for age, education, and other factors. The most strongly associated measure included feelings of indifference toward pregnancy (e.g., "I don't care"). More weakly but still significantly associated measures involved inconsistencies between motivation to avoid pregnancy and anticipated feelings about pregnancy (e.g., "It's important for me to avoid pregnancy right now" but "I would be happy to discover I were pregnant").

Conclusions: The link between pregnancy ambivalence and contraception depends on how ambivalence is measured. Researchers should seek consistency and/or be explicit about their operationalization. Young adults who say they "wouldn't care" if they got pregnant may be especially likely to dispense with contraceptive use.

\section{P-21: Time Trends in Concurrent and Separate Cigarette Smoking and Marijuana Use Among U.S. Reproductive-Aged Women}

Marian Jarlenski (presenting author), ${ }^{1}$ Wyatt Koma, ${ }^{1}$ Jennifer Zank, ${ }^{1}$ Lisa Bodnar, ${ }^{2}$ Debra Bogen, ${ }^{3}$ and Judy Chang ${ }^{4}$

${ }^{1}$ Department of Health Policy and Management and ${ }^{2}$ Department of Epidemiology, University of Pittsburgh Graduate School of Public Health; and ${ }^{3}$ Department of Pediatrics and ${ }^{4}$ Department of Obstetrics, Gynecology \& Reproductive Sciences, Magee-Womens Hospital

Background and Objectives: Studies of marijuana use and women's reproductive health may be confounded by concurrent cigarette use. Trends in the prevalence of cigarette and marijuana use among women are unknown. We investigated time trends in the prevalence of concurrent and separate cigarette and marijuana use among U.S. reproductive-age women.

Methods: We used National Survey of Drug Use and Health (2005-2015) data on female respondents ages 18-44 years. We calculated time trends in the prevalence of past 30-day use of cigarette only, marijuana only, or concurrent cigarette and marijuana use, adjusting for age, race, and education. We also calculated adjusted time trends in the prevalence of women reporting that they perceived "no risk" from using marijuana once or twice a week. We conducted analyses among the full sample and among pregnant women. 
Results: From 2005-2015, the adjusted prevalence of women using only cigarettes declined from $24.5 \%$ to $19.9 \%$, and the adjusted prevalence of women using only marijuana increased from $1.8 \%$ to $4.2 \%$. The adjusted prevalence of concurrent cigarette and marijuana use was stable over time (5\%). Among pregnant women, the adjusted prevalence of marijuana-only use increased from $0.3 \%$ to $1.0 \%$. In 2015 , an adjusted $17.8 \%$ of women reported that smoking marijuana once or twice a week posed "no risk," a fourfold increase from 2005.

Conclusions: The proportion of U.S. reproductive-age women use marijuana without smoking cigarettes doubled from 2005-2015. It is important to investigate the independent effects of marijuana use and interactions between cigarette and marijuana use on reproductive health.

\section{P-22: Pioglitazone, a Peroxisome Proliferator-Activated Receptor Gamma (PPARG) Agonist, Protects Against Altitude-Associated Fetal Growth Restriction in Mice}

Colleen Glyde Julian (presenting author), Gabriel Wolfson, and David A. Schwartz

Department of Medicine, University of Colorado Anschutz Medical Campus

Background and Objectives: The hypoxia of high altitude impairs maternal vascular adaptation to pregnancy and reduces fetal growth. Peroxisome proliferator-activated receptor gamma (PPARG) mediates the transcription of numerous genes that are pivotal for placentation and normal vascular function during pregnancy. The PPARG pathway is repressed by hypoxia, in cases of preeclampsia and/or intrauterine growth restriction and during high-altitude pregnancy. PPARG expression also is directly related to fetal and placental weight. Our objective was to determine whether treatment with a PPARG agonist (pioglitazone [PIO]) during high-altitude pregnancy reduces the magnitude of altitude-associated fetal growth restriction in mice.

Methods: Pregnant C57/BL6 dams were randomly assigned to receive either a PPARG agonist $(0.2 \%$ Actos containing $0.05 \%$ PIO) or control diet under normoxic (Norm) or hypoxic (Hx) conditions. Hyperbaric $\left(\mathrm{P}_{\mathrm{B}} 760 \mathrm{mmHg}\right)$ and hypobaric $\left(\mathrm{P}_{\mathrm{B}}\right.$ $460 \mathrm{mmHg}$ ) chambers were used for Norm and Hx exposures, respectively. Experimental diets and exposures began on gestational day (GD) 14.5 and continued until GD 18.5.

Results: PIO treatment reduced the magnitude of hypoxiainduced fetal growth restriction $(P<0.01)$. In controls, Hx reduced fetal weight $21 \%(P<0.001)$, compared to an $8.5 \%$ reduction in PIO-treated animals $(P<0.05)$. As a result, under Hx conditions, PIO-treated mice weighed $16.5 \%$ more than controls $(P<0.001)$. Hx exposure reduced placental weight and fetal number; however, this effect was equivalent in PIO-treated animals and controls.

Conclusions: Dietary supplementation with a PPARG agonist during pregnancy reduces the magnitude of hypoxia-induced fetal growth restriction in mice.

\section{P-23: Controlling for Contaminants in Low-Biomass Microbiome Experiments}

Lisa Karstens (presenting author), ${ }^{1}$ Mark Asquith, ${ }^{2}$ Rahel Nardos, ${ }^{2}$ Tom Gregory, ${ }^{2}$ Damien Fair, ${ }^{3,4}$ Jim Rosenbaum, ${ }^{5}$ Johnathan Braun, ${ }^{6}$ and Shannon McWeeney ${ }^{1}$
${ }^{1}$ Department of Medical Informatics and Clinical Epidemiology, ${ }^{2}$ Department of Obstetrics and Gynecology, ${ }^{3}$ Department of Behavioral Neuroscience, ${ }^{4}$ Department of Psychiatry, and ${ }^{5}$ Division of Arthritis \& Rheumatic Diseases, Oregon Health \& Science University; ${ }^{6}$ Department of Pathology and Laboratory Medicine, David Geffen School of Medicine, University of California, Los Angeles

Background and Objectives: Advances in genomic sequencing have revolutionized our ability to identify and study microbes. Scientists can now characterize microbial communities-referred to as microbiomes-without depending on culture-based laboratory techniques. These developments have led to an increased desire to understand the role of microbes in environments of low microbial biomass, such as the urinary tract. One challenge that limits our ability to accurately characterize microbial communities is bacterial contamination introduced during sample processing. Here, we present a method to control for contaminants in low biomass samples.

Methods: Urine was collected with a transurethral catheter from women $(n=38)$ as part of an ongoing longitudinal study. To identify laboratory contaminants, a mock community consisting of known bacteria was subject to a series of dilutions. The $16 \mathrm{~S}$ rRNA gene was extracted, amplified, and sequenced with MiSeq. Contaminant profiles were created by separating the non-mock community sequences from the mock community samples. We utilized a Bayesian approach for estimation of the proportion and subsequent removal of contaminants in the samples.

Results: The expected microbial communities from the diluted mock community samples were recovered. Estimates of contamination were obtained from urine samples (range 0-97\%) and removed, leading to a more accurate representation of the microbial communities residing in the bladder.

Conclusions: Inclusion of contaminating bacteria in analyses can lead to incorrect conclusions of microbial composition and function. Using a dilution series of mock communities, it is possible to computationally identify and remove laboratory contaminants from low biomass samples.

\section{P-24: No Substantial Sex Differences in Tenofovir Exposure After Directly Observed Dosing of TDF/FTC for HIV Pre-Exposure Prophylaxis as Assessed via Hair Concentrations}

Catherine A. Koss (presenting author), ${ }^{1}$ Albert Y. Liu, ${ }^{3}$ Peter Bacchetti, ${ }^{2}$ Samantha MaWhinney, ${ }^{4}$ Cricket McHugh, ${ }^{4}$ Karen Kuncze, ${ }^{1}$ Alexander Louie, ${ }^{1}$ Sharon Seifert, ${ }^{4}$ Howard Horng, ${ }^{1}$ Monica Gandhi, ${ }^{1}$ and Peter L. Anderson ${ }^{4}$

${ }^{1}$ Department of Medicine and ${ }^{2}$ Department of Epidemiology and Biostatistics, University of California, San Francisco; ${ }^{3}$ Bridge HIV, San Francisco Department of Public Health; ${ }^{4}$ Department of Pharmaceutical Sciences, University of Colorado Anschutz Medical Campus

Background and Objectives: Pre-exposure prophylaxis (PrEP) with tenofovir (TFV) disoproxil fumarate (TDF)/emtricitabine (FTC) prevents HIV acquisition, but several studies in Africa demonstrated lower effectiveness in women than men. This difference was attributed to poor adherence, although lower TFV levels in vaginal versus rectal mucosa could have contributed. Drug concentrations in hair samples reflect cumulative adherence and exposure, which can impact effectiveness. We evaluated 
whether TFV hair concentrations differ between women and men receiving directly observed dosing of TDF/FTC.

Methods: We compared TFV hair concentrations in low-risk, HIV-uninfected volunteers on directly observed TDF/FTC in Denver and San Francisco (DOT-DBS, NCT02022657). TFV concentrations in the proximal 1.5 centimeters of hair (representing 6 weeks of exposure) were analyzed using validated liquid chromatography/tandem-mass-spectrometry methods. Linear regression models with random-person effects modeled TFV hair concentrations in terms of sex, doses given, and days since last dose.

Results: Among 46 individuals (52\% female), median age was 29 years; $57 \%$ white, $15 \%$ African American, $28 \%$ Hispanic ethnicity. Female participants had similar TFV hair concentrations to males (fold-difference $0.95,95 \%$ CI $0.78-1.15$ ). The fold-difference in TFV levels for female versus male participants did not appreciably change when age (0.96, CI 0.79-1.17), weight $(0.90$, CI $0.73-1.12)$, or race $(0.97$, CI $0.79-1.18)$ were added to the model.

Conclusions: Under directly observed dosing, TFV hair concentrations were similar in women and men, regardless of race, age, and weight. As pharmacokinetic metrics are increasingly used to monitor PrEP, these findings provide guidance for assessing adherence-response relationships via hair concentrations in women and men.

\section{P-25: Gender Differences in the Links Between Racial Discrimination and Mental Health Among Justice-Involved Youth}

Aerika Brittian Loyd (presenting author), ${ }^{1}$ Anna Hotton, ${ }^{2,3}$ Angela Walden, ${ }^{4}$ Geri Donenberg, ${ }^{2,5}$ and Erin Emerson ${ }^{2}$

${ }^{1}$ Department of Educational Psychology, ${ }^{2}$ School of Public Health, ${ }^{3}$ Community Outreach Intervention Projects, Division of Epidemiology and Biostatistics, ${ }^{4}$ Institute for Juvenile Research, and ${ }^{5}$ Healthy Youths Program, University of Illinois at Chicago

Background and Objectives: African-American and Latino youth are overrepresented in the juvenile justice system and experience greater health disparities than White youth. One potentially important contributor to elevated mental and physical health problems among these youths may be racial discrimination (RD). Although RD is linked to mental and physical health challenges, few studies have examined the implications of this social stressor for justice-involved youth of color's mental health. The objectives of this research were to examine possible gender-unique relationships between $\mathrm{RD}$, trauma, emotion regulation, internalizing symptoms, and externalizing behaviors.

Methods: Participants $(n=173 ; 86 \%$ African-American; $45 \%$ girls) were a subset of a larger federally funded randomized controlled trial for whom complete data were available.

Results: Our findings suggest that interpersonal experiences with RD (differential treatment between individuals) are associated with negative mental health outcomes for youth. Youth's experiences of RD were associated with increased internalizing symptoms and externalizing behaviors. Furthermore, girls may be particularly vulnerable and potentially sensitive to the deleterious effects of RD, and these experiences may have consequences for their health and development.

Conclusions: In the long term, poor mental health can impact other health issues, such as substance use, and increased risk for further justice system involvement. Lack of understanding re- garding youth's cultural experiences can contribute to less sustained prevention/intervention effects for justice-involved youth of color. To reduce health disparities that exist for this population, future research also should focus on individual and structural characteristics that promote resilience among girls and boys of color involved in the juvenile justice system.

\section{P-26: Maternal Early Life Adversities and Excess Gestational Weight Gain}

Susan M. Mason (presenting author), ${ }^{1}$ Suzanne Canney, ${ }^{1}$ Lisa Bodnar, ${ }^{3}$ Richard MacLehose, ${ }^{1}$ Melissa Avery, ${ }^{2}$ and Dianne Neumark-Sztainer ${ }^{1}$

${ }^{1}$ School of Public Health and ${ }^{2}$ School of Nursing, University of Minnesota; ${ }^{3}$ School of Public Health, University of Pittsburgh

Background and Objectives: Early life adversities, such as childhood maltreatment victimization and food insecurity, are associated with obesity in adulthood. However, little is known about how exposure to early life adversities relates to women's weight gain during pregnancy, an important outcome that influences the subsequent obesity risks of women and their children. We examined the association of early life adversities with excess weight gain during pregnancy.

Methods: Parous women participating in a longitudinal cohort study of weight-related health that had previously collected data on early life adversities (physical abuse, sexual abuse, emotional abuse, neglect, and food insecurity) were invited to participate in a pregnancy survey asking about prepregnancy weight and pregnancy weight gain. The risk of excess pregnancy weight gain, as defined by the Institute of Medicine, was examined as a function of the number of early life adversities experienced.

Results: Of 162 women sent a survey invitation, 129 completed it $(79.6 \%)$. Women with one early life adversity had a $30 \%$ increased risk of excess pregnancy weight gain, while those with two or more adversities had a 50\% increased risk. Among women who were normal weight prior to pregnancy, there was an $80 \%$ increased risk of excess pregnancy weight gain associated with two or more adversities.

Conclusions: Early life adversities were associated with increased risk of excess pregnancy weight gain. The association of early life adversities with pregnancy weight was particularly strong among normal-weight women; it is possible that pregnancy may be a sensitive period during which previously resilient women may first experience adversity-related weight gain.

\section{P-27: An Intervention Aimed at Improving Decision Role Concordance in Newly Diagnosed Breast Cancer Patients}

Cindy B. Matsen (presenting author), ${ }^{1}$ Angela Fagerlin, ${ }^{2}$ Yizhe $\mathrm{Xu},{ }^{2}$ Angela Presson, ${ }^{3}$ and Kimberly A. Kaphingst ${ }^{4}$

${ }^{1}$ Department of Surgery, ${ }^{2}$ Department of Population Health Sciences, ${ }^{3}$ Department of Internal Medicine, and ${ }^{4}$ Department of Communication, The University of Utah

Background and Objectives: Patients often do not play the role they want in health care decisions. We evaluated a providerbased communication intervention on decision role concordance in breast cancer patients. 
Methods: One hundred new breast cancer patients identified their preferred role in decision-making prior to meeting with their surgeon using the Patient Preferences Scale. In the intervention group ( $\mathrm{IG}, \mathrm{N}=50$ ), surgeons were told of the patient's preferred role and discussed this preference. In the control group $(\mathrm{CG}, \mathrm{N}=50)$, surgeons did not receive this information. After the visit, patients indicated their perceived role and the provider identified the patient role they perceived. Role concordance (exact agreement between preferred and perceived roles) was estimated using Cohen's kappa. Patient and provider perceptions were similarly compared. Role concordance between groups was compared using $85 \%$ CIs, statistical significance defined as no overlap of $85 \%$ CIs.

Results: Preferred role distribution was similar between CG and IG; 1 patient $(2 \%)$ in each preferred that the patient decides, $21(\mathrm{CG}=42 \%), 17(\mathrm{IG}=34 \%)$ preferred that the patient decides considering provider opinion, $23(\mathrm{CG}=46 \%), 27(\mathrm{IG}=54 \%)$ preferred that they decide together, $5(\mathrm{CG}=10 \%), 4(\mathrm{IG}=8 \%)$ preferred that the provider decides considering patient opinion, and 0 (CG), $1(\mathrm{IG}=2 \%)$ preferred that the provider decides. Concordance between patients' preferred roles and perceived roles was moderate, CG k=0.41 (85\% CI: $0.25,0.57)$ and IG k $=0.45(0.30$, $0.60)$ and not significantly different. Concordance between patient and provider perception was low, $\mathrm{CG} \mathrm{k}=0.03(-0.11,0.18)$ and IG $\mathrm{k}=0.19(0.04,0.33)$ and not significantly different.

Conclusions: Discussing the preferred role is important in shared decision-making, but without other interventions, it is not sufficient to improve decision role concordance.

\section{P-28: Associations Between Signs and Symptoms of Androgen Excess with Prediabetes and Diabetes in Reproductive-Aged Hispanic/Latina Women in the Hispanic Community Health Study/Study of Latinos (HCHS/SOL)}

Michelle L. Meyer (presenting author), ${ }^{1}$

Daniela Sotres-Alvarez, ${ }^{2}$ Anne Steiner, ${ }^{3}$ Larry Cousins, ${ }^{4}$

Gregory A. Talavera, ${ }^{5}$ Jianwen Cai, ${ }^{2}$

Martha L. Daviglus, ${ }^{6}$ and Laura R. Loehr ${ }^{7}$

${ }^{1}$ Department of Emergency Medicine, ${ }^{2}$ Department of Biostatistics, ${ }^{3}$ Department of Obstetrics and Gynecology, and ${ }^{7}$ Department of Epidemiology, The University of North Carolina at Chapel Hill; ${ }^{4}$ Children's Specialists of San Diego; ${ }^{5}$ Division of Health Promotion and Behavioral Science, San Diego State University; and ${ }^{6}$ Institute for Minority Health Research, University of Illinois at Chicago College of Medicine

Background and Objectives: Androgen excess in women is associated with cardiometabolic risk factors and diabetes; however, the characterization is incomplete in reproductive-aged women and high-risk groups, such as Hispanic/Latina women. We estimate the association of signs and symptoms of androgen excess with prediabetes and diabetes among reproductive-aged Hispanic/Latina women.

Methods: This is a cross-sectional analysis of 1,132 reproductiveaged women who attended the ongoing visit 2 (October 2014 September 2017) by December 2016 of HCHS/SOL, a U.S. community-based cohort study of Hispanic/Latino adults. Signs and symptoms of androgen excess included menstrual cycle irregularities, self-reported polycystic ovary syndrome (PCOS), and oral contraceptive use to regulate menstrual cycles/treat acne. We defined prediabetes and diabetes using fasting glucose, hemoglobin A1c, and self-reported anti-diabetic medication. Odds ratios (OR) and $95 \%$ confidence intervals (CI) were adjusted for study design, demographics, and cardiovascular risk factors.

Results: Signs and symptoms of androgen excess were reported by $28 \%$ (mean age 34 years) and were not significantly associated with prediabetes or diabetes (OR, CI; 1.2, 0.7-2.0). Women reporting menstrual cycle irregularities had a $1.6(\mathrm{CI}$ : 0.7-3.4) times higher odds of prediabetes compared with women reporting regular cycles, and women self-reporting PCOS had a 1.7 (CI: 0.7-4.0) times higher odds of diabetes compared to those who did not, although not statistically significant.

Conclusions: Signs and symptoms of androgen excess were not significantly associated with prediabetes or diabetes; however, analysis of the complete visit will improve statistical power. Future measurement of androgens will be critical to characterize these relationships.

\section{P-29: Rates of Adult and Childhood Trauma in Atlanta Women's Interagency HIV Study (WIHS) Cohort}

Vasiliki Michopoulos (presenting author), ${ }^{1}$ Sierra Carter, ${ }^{1}$ Igho Ofotokun, ${ }^{2}$ Tanja Jovanovic, ${ }^{1}$ and Gretchen $\mathrm{Neigh}^{3}$

${ }^{1}$ Department of Psychiatry \& Behavioral Sciences and ${ }^{2}$ Department of Medicine, Emory School of Medicine; and ${ }^{3}$ Department of Anatomy \& Neurobiology, Virginia Commonwealth University

Background and Objectives: Low socioeconomic status among African-Americans living within urban environments is associated with high levels of trauma exposure and increased risk for HIV infection. Because trauma exposure increases antiretroviral therapy non-compliance and risky behavior, it is critical to characterize the rates of trauma in people living with HIV. The current study was designed to characterize rates of trauma exposure in a sample recruited from the Women's Interagency HIV Study (WIHS) Atlanta cohort, and to compare these rates to those described previously by the Grady Trauma Project (GTP), a large epidemiological survey of trauma exposure in Atlanta, Georgia.

Methods: Participants were women recruited from the Atlanta WIHS cohort $(n=44)$ and provided informed consent. All subjects participated in a clinical interview conducted by a trained clinician.

Results: $90.9 \%$ of the WIHS cohort reported exposure to trauma in their lifetime, a number greater than the $87 \%$ reported in the larger GTP study. The average number of different types of life-threatening traumatic events endorsed per WIHS participant was $4.2 \pm 3.78$. Rates of childhood trauma also were high in the WIHS samples, as $15.9 \%$ of participants endorse one type of childhood sexual, physical, or emotional abuse; $25.0 \%$ endorsed two types of child abuse; and $11.4 \%$ endorsed all three types of childhood trauma. These rates of childhood trauma exposure in the WIHS cohort $(52.3 \%)$ were greater than those reported in the GTP sample (29.4\%).

Conclusions: Overall, these data document levels of adult and childhood trauma exposure in the WIHS cohort that are greater than previously reported rates in a community sample in Atlanta.

\section{P-30: Evaluating Cognitive Behavioral Therapy to Reduce Insomnia, Sedative Hypnotic Use, and Stress in Appalachia Women Ages 45+}

Mairead Eastin Moloney

Department of Sociology, University of Kentucky 
Background and Objectives: Stress-induced transient sleeplessness is too often diagnosed as insomnia and treated with pharmacologic solutions (sedative hypnotics $[\mathrm{SH}]$ ). $\mathrm{SH}$ pose long-term health risks, including the development of neurocognitive disorders like Alzheimer's disease. These health issues are of particular concern to women ages $45+$ of low socioeconomic status, especially Appalachian women (AW). AW experience multiple social, economic, and psychological stressors, have high rates of insomnia and SH use, and fragmented access to health care. Thus, this population would likely benefit from a community-based, non-pharmacologic insomnia therapy, such as cognitive behavioral therapy for insomnia (CBT-I). The objective of this study is to pilot test in-person and online versions of a well-validated CBT-I program that may interrupt the stresssleeplessness cycle in AW ages 45+.

Methods: Forty participants will be randomly assigned to one of the two intervention groups $(n=20$ per intervention); both versions require once-weekly sessions for 5 weeks. In-person CBT-I groups (4-5 participants per group) will be held at the University of Kentucky's Center for Excellence on Rural Health (UK CEHR). Pre- and post-intervention interviews, surveys, a brief cognition test, and salivary cortisol testing will be used to measure participants' sleep, stress, cognition, medication use, and intervention acceptance.

Results: This study has received IRB approval and the support of the UK CEHR. Data collection will begin in July 2017.

Conclusions: This innovative pilot project is a first step in determining if a scalable, community-accessible, non-pharmacologic intervention may improve sleep, reduce stress and $\mathrm{SH}$ use, and ultimately protect cognitive health in AW ages $45+$.

\section{P-31: Effects of Media Exposure to Conflicting Information About Mammography: Results from a Population-Based Survey Experiment}

Rebekah H. Nagler (presenting author), ${ }^{1}$ Marco C. Yzer, ${ }^{1}$ and Alexander J. Rothman ${ }^{2}$

${ }^{1}$ School of Journalism \& Mass Communication and

${ }^{2}$ Department of Psychology, University of Minnesota

Background and Objectives: There is substantial expert disagreement about the use of mammography to screen for breast cancer. This discord plays out in the media, with conflicting messages about the age at and frequency with which screening should occur. We examine whether media exposure to conflicting information about mammography has adverse cognitive effects.

Methods: We conducted an online survey-based experiment with a population-based sample of U.S. women ages 35-55. Participants $(\mathrm{N}=1,474)$ were randomly assigned either to one of three conditions that differed in the amount of conflicting information about mammography presented in a news story (low, medium, or high) or to a no-exposure control condition. Outcomes included confusion about cancer prevention recommendations (e.g., "It is not clear to me how best to avoid cancer"), backlash toward such recommendations (e.g., "Cancer recommendations should be taken with a grain of salt"), and ambivalence about screening (e.g., "I have mixed feelings about getting a mammogram").

Results: Exposure to conflict significantly increased confusion and backlash. Exposure to conflict also significantly influenced ambivalence, but here we observed a quadratic trend, with ambivalence highest among those in the low- and mediumexposure groups. Those highly exposed to conflict might have been less ambivalent because they were confident in their decision not to get screened: mean mammography intentions were lowest among those in the high-exposure group.

Conclusions: Media exposure to conflicting information about mammography produced confusion, backlash, and ambivalence. These cognitions could, in turn, undermine campaigns/ interventions designed to encourage not only mammography, but also other cancer prevention behaviors.

\section{P-32: The Desire to Avoid Pregnancy Scale: a Psychometric Instrument to Measure Pregnancy Preferences}

Corinne H. Rocca (presenting author), ${ }^{1}$ Lauren J. Ralph, ${ }^{1}$ Heather Gould, ${ }^{1}$ Rana Barar, ${ }^{1}$ Mark Wilson, ${ }^{2}$ and Diana G. Foster ${ }^{1}$

${ }^{1}$ Department of Obstetrics, Gynecology and Reproductive Sciences, University of California, San Francisco; and ${ }^{2}$ Graduate School of Education, University of California, Berkeley

Background and Objectives: Existing approaches to measuring women's pregnancy preferences suffer important limitations, including retrospective assessment, overly simple categories, and presumption that women plan pregnancies in advance. A psychometrically valid scale to prospectively measure the ranges of women's pregnancy preferences is sorely needed. Using a rigorous construct modeling approach, we developed a scale to measure women's conscious and unconscious desire to avoid pregnancy.

Methods: We defined the construct and its domains, developed 60 draft items from existing research, and assessed comprehension through 20 cognitive interviews. We then administered all items in surveys with 405 non-pregnant women in five primary and reproductive health care facilities in Texas, Arizona, and New Mexico in June-September 2016. Using item response theory, the leading statistical paradigm for the development and assessment of psychometric instruments, we reduced the item set and assessed the scale's reliability, internal structure validity, and external validity.

Results: The final scale items fell into three domains: cognitive evaluation of preferences, affective feelings, and anticipated practical consequences. Items illustrated good fit to a unidimensional partial credit model. The internal consistency reliability of the scale was 0.95 ; for the individual domains, reliability was $0.90,0.90$, and 0.79 , respectively. The scale met established criteria for internal validity, including appropriately covering the ranges of women's preferences and correspondence between each item's response categories and overall scale scores. We found no evidence of differential item functioning by age or race/ethnicity, garnering evidence for external validity.

Conclusions: A robust measure is available to prospectively measure desire to avoid pregnancy.

\section{P-33: High n-6 Polyunsaturated Fatty Acids in Human Milk Elevates Adipose Deposition in Infants, and in Mice, Hypomethylates PPAR $\gamma 2$ Increasing Later Life Obesity Risk}

Michael C. Rudolph (presenting author), ${ }^{1}$ Bridget E. Young, ${ }^{2}$ Julie A. Houck, ${ }^{1}$ Matthew R. Jackman, ${ }^{1}$ Patricia G. Webb, ${ }^{1}$ Dominick J. Lemas, ${ }^{2}$ Teri L. Hernandez, ${ }^{2}$ Linda A. Barbour, ${ }^{2}$ Yang IV, ${ }^{3}$ Jacob E. Friedman, ${ }^{2}$ Nancy F. Krebs, ${ }^{2}$ and Paul S. MacLean ${ }^{1}$ 
${ }^{1}$ Division of Endocrinology, Diabetes \& Metabolism, ${ }^{2}$ Department of Pediatrics, Section of Neonatology, and ${ }^{3}$ Division of Biomedical Informatics and Personalized Medicine, University of Colorado, School of Medicine, Anschutz Medical Campus

Background and Objectives: Maternal obesity transmits elevated amounts of omega- 6 polyunsaturated fatty acids (n-6 PUFA). Infant exposure to high n-6 PUFA levels during gestation and breastfeeding, which is introduced by the maternal diet, has been associated with increased pediatric adiposity out to 3 years of age. We determine the molecular mechanisms imparted by a maternal diet rich in n- 6 relative to n-3 PUFA, in conditioning obesity prone adipose in offspring.

Results: The ratio of arachidonic (AA, 20:4 n-6) to docosahexaenoic (DHA, 22:6n-3) plus eicosapentaenoic (EPA, 20:5 $n-3)$ acids (n-6/n-3 PUFA), positively associated with infant accumulation of fat mass and \% body fat $(\mathrm{P}=0.038$ and 0.010$)$. Clinical observations were tested in an established mouse model of reduced n-6/n-3 PUFA ratios and maternal obesity. Obese (OB) dams produced significantly greater milk fat $(\mathrm{P}=0.01)$, and fat-1 transgenic expression significantly increased milk n-3 PUFA relative to wildtype dams $(P<0.003)$. Greater n-3 PUFA exposure in offspring of $\mathrm{OB}$ dams led to less total fat mass accumulation. Importantly, enriched n-3 PUFA increased DNA methylation of peroxisome proliferator-activated receptor gamma 2 (PPAR $\gamma 2$ ) to suppress its levels and multiple PPAR target genes in offspring adipose, leading to smaller but more numerous mature adipocytes and a greater population of adipocyte precursor cells. High levels of perinatal n-3 PUFA protected adults against diet-induced obesity with better glucose clearance.

Conclusions: Cumulatively, our results indicate that more n-3 PUFA (with less n-6 PUFA) early in life, regulates adipogenesis, potentially by establishing a healthier DNA methylation pattern (epigenetics), to improve metabolic response to an adult obesogenic diet.

\section{P-34: Identification of Genomic Markers of Atherosclerosis in HIV-Infected Women}

Sandra E. Safo (presenting author), ${ }^{1}$ Anandi Sheth, ${ }^{2}$ Arshed Quyyumi, ${ }^{3}$ Lance Waller, ${ }^{1}$ and Qi Long ${ }^{1}$

${ }^{1}$ Department of Biostatistics and Bioinformatics, Emory University; and ${ }^{2}$ Division of Infectious Diseases and ${ }^{3}$ Division of Cardiology, Emory School of Medicine

Background and Objectives: Cardiovascular diseases (CVD) are one of the leading co-morbidities in HIV patients. Carotid intima-media thickness (CIMT) is used as a marker of early atherosclerosis (ASCVD). CVD risk prediction models use traditional risk factors only, but there is evidence of genomic contribution to CIMT in HIV-uninfected population. It is important to find genetic markers that increase subclinical CVD risk for targeted therapeutic CVD interventions in HIV-infected women. We seek to understand how genetic markers modulate traditional risk factors and HIV parameters to increase CIMT risk in HIV-infected women.

Methods: Data (training: $\mathrm{N}=831,419 \mathrm{HIV}-, 412 \mathrm{HIV}+$, validation: $\mathrm{N}=830,416$ HIV-, $414 \mathrm{HIV}+$ ) were obtained from women in the Women's Interagency HIV Study with CIMT measured. We used a dimension reduction and biomarker selection method, sparse linear discriminant analysis, on training data to identify genetic markers differentiating high- versus low- risk CIMT. We assessed clinical utility of single nucleotide polymorphisms (SNPs) (identified in first step) above traditional CVD and HIV risk factors using AUC from multivariable logistic regression models on validation data.

Results: We identified two SNPs, rs15285 and rs4684104, that improved likelihood of detecting high-risk CIMT above known risk factors in HIV-infected women (AUC $0.77 \uparrow 0.79, P$ value $<0.001)$ but worsened predictive ability in HIV-uninfected women $(0.86 \downarrow 0.74, P$-value $<0.001)$.

Conclusions: SNPs rs15285 and rs4684104 seem to offer protection against ASCVD in HIV-uninfected women, but not in HIV-infected women. These SNPs lie respectively in the LPL and PPARG gene regions and their implications should be explored in different subgroups of HIV-infected women.

\section{P-35: Pregnancy but Not Sex Impacts Lassa Fever Outcome}

John S. Schieffelin (presenting author), ${ }^{1}$ Jeffrey Shaffer, ${ }^{2}$ Marie Krousel-Wood, ${ }^{3}$ Robert F. Garry, ${ }^{4}$ and Donald S. Grant ${ }^{5}$

${ }^{1}$ Department of Pediatrics, ${ }^{3}$ Department of Medicine, and ${ }^{4}$ Department of Microbiology and Immunology, Tulane University School of Medicine; ${ }^{2}$ Department of Biostatistics and Bioinformatics, Tulane University School of Public Health and Tropical Medicine; and ${ }^{5}$ Kenema Government Hospital, Sierra Leone

Background and Objectives: Lassa fever (LF), a highly fatal viral hemorrhagic fever (VHF) caused by Lassa virus (LASV) in the family Arenaviridae, is endemic to West Africa. The objective of this study was to identify sex differences in the clinical presentation and outcome of hospitalized Lassa fever patients.

Methods: Patients were enrolled in an observational study of suspected Lassa fever patients conducted at the Lassa Fever Ward of the Kenema Government Hospital (KGH LFW) in Kenema, Sierra Leone between January 2010 and December 2016. All patients presenting to the ward and meeting the case definition for suspected LF were eligible for enrollment. Confirmed LF patients were admitted to the ward for disease-specific therapy. Admission and daily signs and symptoms were recorded on standardized report forms. Blood samples were collected for analysis at presentation. The primary outcome was defined as survival at hospital day 10 .

Results: There were 188 Lassa antigen positive and $510 \operatorname{IgM}$ positive patients admitted to the KGH LFW during the study period. Fifty-five percent (390) were female. The Ag+ patients were slightly younger than the IgM+ patients ( 22 vs. 25 years, $\mathrm{P}=.02$ ). No sex differences were seen in the presenting characteristics or outcome. Overall mortality rate was $44 \%$. Among women between the ages of 15 and 50 years, 35\% (25) were pregnant. Pregnant women were 2.62 times more likely to die than non-pregnant women $(61 \%$ vs. $38 \% ; \mathrm{P}=.01)$.

Conclusions: A higher index of suspicion for LF is needed among pregnant women in endemic areas.

\section{P-36: Sex Differences in Knee Forces and Moments that Occur with Anterior Cruciate Ligament Rupture with Cadaveric Impact Simulations}

Nathan D. Schilaty (presenting author), Nathaniel A. Bates, Christopher V. Nagelli, Aaron J. Krych, and Timothy E. Hewett

Department of Orthopedic Surgery, Mayo Clinic 
Background and Objectives: Females have an increased risk of injury to the anterior cruciate ligament (ACL), compared to male counterparts. Seventy-five percent of ACL injuries are noncontact mechanism, demonstrating that biomechanical kinetics/kinematics are significant risk factors. This study was designed to determine mechanisms of knee loading that cause ACL failure during a common athletic task-a drop vertical jump (DVJ). We hypothesized that female limbs would demonstrate differences in knee forces/moments that predispose females to ACL injury, compared to males.

Methods: A custom-designed cadaveric impactor simulated DVJs in lower extremities of 35 specimens (22M:13F; ages 2452). Pneumatic cylinders applied in vivo determined knee forces/ moments to the tibia and a gravity-driven drop sled of 0.5 body weight was released from a height of $31 \mathrm{~cm}$ with quadriceps and hamstrings co-contracted (1:1 ratio). Multi-axial load cells measured forces/moments of the knee.

Results: Females demonstrated an increase of maximum knee compression $\left(\mathrm{F}_{1,24}=6.6497 ; P=0.0168\right)$ and maximum knee extension moment $\left(\mathrm{F}_{1,24}=8.1078 ; P=0.0091\right)$. Normalization of maximum values by body weight/height demonstrated increased knee abduction moment $(\mathrm{KAM})\left(\mathrm{F}_{1,24}=9.1274 ; P=0.0090\right)$ of females, compared to males. At $100 \mathrm{msec}$ post-impact, females demonstrated increased knee extension moment $\left(\mathrm{F}_{1,24}=14.1998 ; P=0.0010\right)$ and $\operatorname{KAM}\left(\mathrm{F}_{1,24}=6.6006 ; \mathrm{P}=0.0172\right)$, compared to males.

Conclusions: Knee extension and KAM are theorized mechanisms that load the ACL and consequently lead to rupture. Females exhibit greater forces/moments at the knee that predispose them to ACL injury, compared to males.

\section{P-37: Increased Nonexudative Age-Related Macular Degeneration Diagnosis Among Medicare Beneficiaries with Rheumatoid Arthritis}

Gloriane Schnabolk (presenting author), ${ }^{1}$ Bärbel Rohrer, ${ }^{1,2}$ and Kit Simpson ${ }^{3}$

${ }^{1}$ Department of Ophthalmology and ${ }^{3}$ Department of Healthcare Leadership and Management, Medical University of South Carolina; and ${ }^{2}$ Research Service, Ralph H. Johnson VA Medical Center

Background and Objectives: Age-related macular degeneration (AMD) is the leading cause of blindness in the United States. The role of secondary inflammatory disease on AMD progression is largely unknown. This study investigates the association between AMD and rheumatoid arthritis (RA), two diseases more common in women, using MarketScan ${ }^{\circledR}$ data for patients $\geq 65$ years on Medicare.

Methods: Baseline data were extracted for subjects with at least two ICD-9 diagnosis codes of RA and control subjects with no $\mathrm{RA}$, and were matched at baseline by propensity score. Matched cohort data were extracted for post-baseline time and examined up to 4.5 years of follow-up for ICD-9 diagnosis code AMD records. Multivariable logistic regression and Fisher's exact test were used to compare odds of AMD diagnosis post-baseline for RA subjects and matched controls. Survival analysis was used to examine days until first AMD diagnosis between RA patients and controls.

Results: Risk of new AMD diagnosis was elevated in RA patients (OR of 2.08 [95\% CI 1.98-2.18]), with an increase in nonexudative AMD patients $(P<.0001)$. Risk was elevated in female $(n=27,548)$ patients (OR of 1.11 [95\% CI 1.05-1.17]) when compared to males $(n=9,704 ; P<0.001)$. The time to first AMD diagnosis was shorter for RA subjects than controls $(P<.0001)$.
Conclusions: Our analysis provides further support of association between RA diagnosis and increased AMD diagnosis. These data might support the hypothesis that synergistic effects of systemic inflammation exist such as those generated by RA and local inflammation in the eye, which together will increase the risk for AMD development.

\section{P-38: The Integration of Health Behavior Change Theory into the Design of a mHealth Intervention to Improve Eating Behaviors among Overweight and Obese African American Men and Women}

Jylana L. Sheats (presenting author), ${ }^{1}$

Revonda M. Darensbourg, ${ }^{2}$ Donald D. Rose, ${ }^{1}$

Lydia Bazzano, ${ }^{3}$ and Patrick Bordnick ${ }^{4}$

${ }^{1}$ Global Community Health \& Behavioral Sciences Department, ${ }^{2}$ Tulane Prevention Research Center, and ${ }^{3}$ Department of Epidemiology, Tulane University School of Public Health \& Tropical Medicine; and ${ }^{4}$ School of Social Work, Tulane University

Background and Objectives: Although there has undoubtedly been a surge in diet-related mHealth interventions, limited research has been on African-Americans (AA). Among overweight/obese AA adults ages 30+ years owning a cellular/ smartphone, aims were to (1) assess the acceptability of mHealth for healthy eating (HE) support; (2) examine salient consequences, circumstances, and referents for HE; and (3) identify appealing features for HE mHealth interventions.

Methods: Participants were recruited from New Orleans-area health clinics. Consenting individuals completed the study survey and attended a focus group modeled after Theory of Planned Behavior (TPB) belief elicitation processes assessing salient consequences, circumstances, and referents for HE. Participants also described "wish-list" features of an HE mHealth intervention. Descriptive statistics/content analyses were conducted to describe/organize data.

Results: Participants $(N=16)$ were primarily women $(88 \%)$ and were ages 35 to 78 years. All expressed interest in receiving text or app-based HE support. Salient beliefs were similar for both sexes. Exemplary advantages of HE were better health and maintaining independence; looking ill/drugged from associated weight-loss was a disadvantage (consequences). Health improvements and encouragement made HE easier; whereas New Orleans food/culture and contraindications made it difficult (circumstances). Family and health care providers were salient referents. Receiving two or fewer messages/day, bigger keys, one- and two-way messaging, goal-setting, and messages framed around "improving health" versus weight-loss were "wish-list" preferences.

Conclusions: Overall, mHealth-based support for HE was acceptable to participants. mHealth interventions that consider salient beliefs and end-user preferences in the design process are needed and may be beneficial for improving AA healthy eating behaviors.

\section{P-39: Using Digital Health to Improve Adherence to the DASH Diet Among Women at Risk for Cardiovascular Disease}

Dori Steinberg (presenting author), ${ }^{1}$ Laura Svetkey, ${ }^{2}$ and Gary Bennett ${ }^{3}$

${ }^{1}$ Duke Global Health Institute, ${ }^{2}$ Department of Medicine, and ${ }^{3}$ Department of Psychology, Duke University 
Background and Objectives: The Dietary Approaches to Stop Hypertension (DASH) diet is proven to reduce blood pressure and cardiovascular disease (CVD) risk, yet adherence to DASH is poor. Innovative approaches, such as digital health, are needed to improve DASH uptake, particularly among women who experience disproportionate CVD burden. Our objective was to determine if a weight loss intervention that included healthy diet goals and digital health would improve weight, blood pressure, and DASH adherence among high-risk women.

Methods: Women were enrolled in a 12-month randomized controlled trial testing a weight loss intervention that included mobile technologies and provider counseling. The Block Food Frequency Questionnaire assessed diet at baseline and 12 months. DASH adherence scores (range 0-9) were calculated.

Results: Women $(n=107)$ were on average age 52.3 years and obese (BMI: $\left.36.5 \mathrm{~kg} / \mathrm{m}^{2}\right)$. Most $(60 \%)$ were black and low-income $(\leq \$ 25,000 / y) ; 80 \%$ had hypertension. On average, the intervention produced clinically meaningful changes in weight $(-3.6 \mathrm{~kg} \pm 6.5$; $\mathrm{P}<.001)$, but not blood pressure. At baseline, DASH scores were low $($ mean $=3.57 \pm 1.15)$ and $80 \%$ had a score of $\leq 4.5$. DASH scores did not improve over time [mean difference $(95 \% \mathrm{CI})=-0.09(-.34$, .17)]. However, improvements in DASH were correlated with lower $\mathrm{BP}(r=-.20 ; \mathrm{P}=.05)$. Hypertensive women had significantly lower DASH scores at 12 months compared to non-hypertensive women (3.24 \pm 1.3 vs. $4.03 \pm 1.8 ; \mathrm{P}=.029)$.

Conclusions: A weight loss intervention that included digital health components produced weight loss but did not improve DASH scores or blood pressure among high-risk women. To reduce CVD burden, digital health interventions that specifically target DASH adherence are needed.

\section{P-40: Opioid Use Disorder in Pregnant Women in Jails and Prisons}

Carolyn Sufrin (presenting author), ${ }^{1,2}$ Lauren Beal, ${ }^{1}$ Mary Peeler, ${ }^{3}$ William Mosher, ${ }^{4}$ and Jennifer Clarke ${ }^{5,6,7}$

${ }^{1}$ Department of Gynecology and Obstetrics, ${ }^{2}$ Department of Health, Behavior and Society, ${ }^{3}$ Johns Hopkins School of Medicine; and ${ }^{4}$ Department of Population, Family, and Reproductive Health, Johns Hopkins Bloomberg School of Public Health; ${ }^{5}$ Department of Medicine and ${ }^{6}$ Department of Obstetrics and Gynecology, Warren Alpert Medical School of Brown University; and ${ }^{7}$ Rhode Island Department of Corrections

Background and Objectives: Numerous intersections exist between the opioid epidemic and the criminal justice system, from drug-related crimes to the health needs of incarcerated people with opioid use disorder (OUD). Most of the 210,000 incarcerated women in the United States are reproductive-aged, and some enter jail or prison pregnant. Medication-assisted treatment (MAT) is the established standard in pregnancy, as detoxification has maternal and fetal risks. It is not known how many incarcerated pregnant women have OUD or what treatment they receive. We collected national prevalence data on OUD among pregnant incarcerated women and describe existing policies of management of OUD in pregnancy at jails and prisons.

Methods: We collected monthly data for 6 months from 20 state prisons and four jails on OUD in pregnancy from sites participating in the Pregnancy in Prison Statistics (PIPS) surveillance project. All 22 prisons and six jails in PIPS reported OUD policies.

Results: Four prisons (18\%) and two jails (33\%) do not provide MAT and compel pregnant women with OUD to detox.
Of sites with MAT, most provide methadone only (prisons $=55 \%$, jails $=50 \%$, while some offer methadone and buprenorphine (prisons $=28 \%$, jails $=25 \%$ ); three prisons and one jail provide buprenorphine only. Twenty-one percent of pregnant women admitted to prisons and $17 \%$ to jails had OUD. Of these women, $31 \%$ in prisons and $50 \%$ in jails were detoxed.

Conclusions: Pregnant incarcerated women with OUD do not consistently receive MAT, despite established guidelines to avoid withdrawal in pregnancy. Interventions are needed to ensure MAT access, optimizing outcomes for this vulnerable population of pregnant women.

\section{P-41: Excess Cost of Cervical Cancer Screening in Women at the Extremes of Age and Post-Hysterectomy Within a Single Institution}

Deanna Teoh (presenting author), ${ }^{1}$ Gretchen Hultman, ${ }^{2}$ McKenzie DeKam, ${ }^{1}$ Minnu Monu, ${ }^{1}$ Rachel Isaksson Vogel, ${ }^{1,3}$ Levi Downs, ${ }^{1}$ Melissa A. Geller, ${ }^{1}$ Chap Le, ${ }^{4}$

Genevieve Melton-Meaux, ${ }^{2,5}$ and Shalini Kulasingam ${ }^{6}$

${ }^{1}$ Department of Obstetrics, Gynecology \& Women's Health, ${ }^{2}$ Institute for Health Informatics, ${ }^{3}$ Biostatistics \& Bioinformatics, Masonic Cancer Center, ${ }^{4}$ School of Public Health, Division of Biostatistics, ${ }^{5}$ Department of Surgery, and ${ }^{6}$ Department of Epidemiology \& Community Health, University of Minnesota

Background and Objectives: Overscreening and overdiagnosis are associated with excess costs that do not result in improved health outcomes. We estimate the excess cost of nonindicated cervical cancer screening at the extremes of age or post-hysterectomy for women in a single health care system.

Methods: All non-indicated Pap tests performed from September 1, 2012, to August 31, 2014, in women $<21,>65$ years old or post-hysterectomy at our institution were identified. Costs were obtained from a literature review and Centers for Medicare $\&$ Medicaid Services Medicare and Medicaid data and applied to the non-indicated tests, assuming management of abnormal results per national guidelines.

Results: In the $<21$ year-old group, there were 259 nonindicated Pap tests, with $35(13.5 \%)$ abnormal results. The cost for initial screening and diagnostic tests was $\$ 25,406$, with an additional $\$ 4,291$ over 2 years for follow-up testing. In the $>65$ year-old group, there were 536 non-indicated Pap tests performed, with $14(2.6 \%)$ abnormal results. The initial cost was $\$ 51,858$, with an additional $\$ 2,520$ over 5 years for follow-up testing. In the post-hysterectomy group, there were 597 nonindicated Pap tests performed, with 39 (6.5\%) abnormal results. The initial cost was $\$ 60,904$, with an additional $\$ 15,260$ over 5 years for follow-up testing. In the entire group, there were only 2 $(0.1 \%)$ diagnoses of high-grade dysplasia (VAIN3).

Conclusions: Non-indicated cervical cancer screening over a 5 -year period in women at the extremes of age and posthysterectomy resulted in excess costs exceeding $\$ 160,000$ for screening, diagnostic tests, and follow-up, with minimal improvement in detection of high-grade dysplasia.

\section{P-42: Strengthening Maternity Care by Identifying Factors Associated with Longer Latent Labor}

Ellen L. Tilden (presenting author), ${ }^{1,2}$ Mekhala Dissanayake, ${ }^{2}$ Jonathan M. Snowden, ${ }^{2,3}$ and Aaron B. Caughey ${ }^{2}$ 
${ }^{1}$ School of Nursing, ${ }^{2}$ Department of Obstetrics and Gynecology, and ${ }^{3}$ School of Public Health, Oregon Health \& Science University

Background and Objectives: Latent labor is defined as the onset of labor to the onset of active labor, and it generally occurs outside of the hospital. Most latent labor studies define latent labor onset via clinician judgment or timing of hospital admission; this likely truncates estimated latent labor lengths. Additionally, few studies examine variables associated with latent labor length. The purpose of this study was to: (1) describe latent labor in a sample of low-risk women in spontaneous labor using the woman's selfidentification of latent labor onset, and (2) determine the association between maternal demographic/health/labor characteristics and length of latent labor at the mean, $80 \%, 90 \%$, and $95 \%$ percentiles.

Methods: Descriptive statistics identified latent labor length mean and percentiles as well as sample sizes of those continuing latent labor after the mean and each percentile. Analysis of characteristics associated with latent labor lengths were tested using chi-squared tests of independence and Mann-Whitney tests. Analyses were stratified by parity.

Results: Characteristics significantly correlated with longer latent labor in this entire sample, regardless of parity, included participating in childbirth education, chorioamnionitis, and fetal malposition at delivery. A characteristic uniquely correlated with longer latent labor among nulliparous women was first trimester prenatal care initiation and a minimum of five visits. A characteristic uniquely correlated with longer latent labor among multiparous women was participation in group prenatal care.

Conclusions: Longer latent labor was associated with several maternal demographic/health/labor characteristics. Associations were unique to parity but shared common patterns.

\section{P-43: Hyperandrogenemia Is Common in Asymptomatic Women and Is Associated with Increased Metabolic Risk}

Laura C. Torchen (presenting author), ${ }^{1}$ Margrit Urbanek, ${ }^{2}$ and Andrea Dunaif ${ }^{3}$

${ }^{1}$ Division of Endocrinology, Ann \& Robert H. Lurie Children's Hospital of Chicago, Feinberg School of Medicine, and

${ }^{2}$ Division of Endocrinology, Metabolism and Molecular Medicine, Northwestern University; and ${ }^{3}$ Division of Endocrinology, Diabetes, and Bone Diseases, Icahn School of Medicine at Mount Sinai

Background and Objectives: Pre- and postmenopausal women with metabolic syndrome (MetS) have higher testosterone levels than unaffected women. Hyperandrogenemia (HA) is also common in obese girls. We aimed to test the hypothesis that $\mathrm{HA}$ is a marker for increased cardiometabolic risk in wellphenotyped women with regular menses.

Methods: We screened 168 premenopausal women with regular menses and no clinical hyperandrogenism as potential control subjects (eumenorrheic, EM). Anthropometric measurements, reproductive hormones, fasting, and post-glucose challenge metabolic parameters were compared to 132 women with PCOS.

Results: Twenty-seven percent $(n=46)$ of EM had HA. MetS risk was increased in HA-EM (adjusted odds ratio [AOR] 2.8, CI 1.1-7.2) and PCOS (AOR 5.2, CI 2.4-11.5) compared to NA-EM (normoandrogenemic, eumennorheic). Risk of IGT and IFG did not differ in HA-EM versus NA-EM, while PCOS had increased risk of IGT compared to NA-EM (AOR 4.3, CI 1.8-
10.6). SHBG levels were lower in HA-EM compared with both NA-EM $(\mathrm{P}=0.0007)$ and PCOS $(\mathrm{P}<0.0001)$. There was no association between SHBG genetic polymorphisms robustly associated with protein levels (rs 1799941, rs6275, rs625) and SHBG levels in our cohort. Eighteen HA-EM women had high AMH levels (39\%), suggesting these women may fulfill Rotterdam PCOS criteria. The increased risk for MetS persisted after excluding HA-EM with high AMH.

Conclusions: HA was common in EM without clinical hyperandrogenism. HA-EM with high AMH levels may have fulfilled Rotterdam PCOS criteria. MetS risk was higher in HA-EM than NA-EM, but highest in PCOS. HA may be a biomarker of cardiometabolic risk in asymptomatic women.

\section{P-44: The Influence of Sex and Diazepam on Hippocampal Neurogenesis Following Traumatic Brain Injury}

Laura E. Villasana

Department of Anesthesiology and Perioperative Medicine, Oregon Health Science University

Background and Objectives: In response to a traumatic brain injury (TBI), the brain increases its generation of new neurons (neurogenesis) within the hippocampus, a region critical for memory. Because constitutive neurogenesis is important for learning, post-traumatic neurogenesis may be important for cognitive recovery. Studies suggest that women have worse cognitive outcomes following TBI, including greater impairments in memory and mood, which are domains that are sensitive to changes in neurogenesis. Therefore, sex may influence post-traumatic neurogenesis. Additionally, the type of sedatives used during TBI care also might influence post-traumatic neurogenesis. The objective was to determine whether there are sex differences in post-traumatic neurogenesis and if sex modulates the potential effects of diazepam (a clinically relevant sedative) on post-traumatic neurogenesis.

Methods: Adult male and female wild-type mice underwent a controlled cortical impact injury (CCI) or sham treatment. Posttraumatic neurogenesis was assessed 2 weeks later. To determine whether proliferation of new neurons account for sex differences in post-traumatic neurogenesis and to determine whether diazepam influences this response, mice underwent CCI or sham treatment with or without diazepam. Mice were sacrificed 3 days later and assessed for proliferation using the mitotic marker BrdU.

Results: Two weeks after CCI, post-traumatic neurogenesis was greater in female mice. Three days after CCI, mice had a greater density of BrdU positive (+) cells independent of sex. However, diazepam inhibited CCI-induced increases in BrdU+ cells in male but not female mice.

Conclusions: Survival versus proliferation of new neurons may underlie sex differences in post-traumatic neurogenesis. Furthermore, our data indicates sex differences in how diazepam affects proliferation after TBI.

\section{P-45: A Pilot Study of Exome Sequencing (ES) of Cell Free Fetal DNA (cff DNA) for Detection of Pathogenic Fetal Variants}

Neeta L. Vora (presenting author), ${ }^{1}$ Kelly Gilmore, ${ }^{1}$ Emily Hardisty, ${ }^{1}$ Kim Boggess, ${ }^{1}$ and Piotr Mieczkowski ${ }^{2}$

${ }^{1}$ Department of Obstetrics and Gynecology and ${ }^{2}$ High Throughput Sequencing Facility, The University of North Carolina at Chapel Hill 
Background and Objectives: Detection of single gene disorders and copy number variants via cff DNA remains challenging. Our objective was to perform exome sequencing (ES) on extracted cff DNA from women with fetuses affected with single gene disorders or microdeletion syndromes.

Methods: Patients carrying a fetus with a known single gene disorder or microdeletion syndrome, as confirmed by genetic testing performed on fetal specimens, were approached for participation. Results are available on two thirds of enrolled patients. In case 1, there was a deletion of exon 1 in the ATP7A gene known to cause Menke's disease (X-linked recessive) in the hemizygous state. In case 2, there was a homozygous pathogenic variant in $\mathrm{P} 3 \mathrm{H} 1$ gene causing osteogenesis imperfecta, type VIII (c. 1120G >T [p. Glu374Ter]). cf DNA was extracted from plasma and DNA ES libraries were prepared. Paired end ES of libraries was performed on HiSeq4000 system (200x coverage). Data analysis was performed by investigators blinded to the known fetal results.

Results: The homozygous pathogenic variant in $\mathrm{P} 3 \mathrm{HI}$ gene was identified via ES of the cf DNA. We estimated the frequency of fetal homozygous and heterozygous variants using the maternal sample only. We were unable to detect the deletion in the ATP7A gene.

Conclusions: ES of cf DNA detected in maternal blood can identify pathogenic variants in fetuses with single gene disorders. Noninvasive interrogation of the fetal exome increases the ability to identify X-linked disorders and obtain genotype/phenotype information prenatally. Use of this technology may increase our understanding of developmental differences between genders.

\section{P-46: The Impact of Paternal Childhood Adversity on Offspring Neurodevelopmental and Behavioral Disorders}

Roy Wade, Jr. (presenting author), ${ }_{5}^{1}$ Marisa Brant, ${ }^{1}$ Mary D. Sammel, ${ }^{2}$ Tracy L. Bale, ${ }^{5}$

C. Neill Epperson, ${ }^{3}$ and Scott A. Lorch ${ }^{4}$

${ }^{1}$ Department of Pediatrics, The Children's Hospital of Philadelphia, ${ }^{2}$ Department of Biostatistics and Epidemiology, ${ }^{3}$ Department of Psychiatry, and ${ }^{4}$ The Children's Hospital of Philadelphia, Perelman School of Medicine, University of Pennsylvania; and ${ }^{5}$ Department of Biomedical Sciences, School of Veterinary Medicine, University of Pennsylvania

Background and Objectives: Adverse childhood experiences (ACE), such as childhood abuse, neglect, and exposure to household stressors have been associated with childhood neurodevelopmental and behavioral disorders (NDBD), including developmental delay, behavioral problems, cognitive impairment, and attention-deficit/hyperactivity disorder. Preclinical studies have demonstrated a sex-specific link between parental stress and offspring health. Clinical studies investigating the transgenerational transmission of ACE-effects have largely focused on maternal ACE, despite epidemiological studies illustrating the importance of paternal stress on offspring NDBD risk. As a result, we know little about the influence of paternal ACE on offspring NDBD risk. We examine the impact of paternal ACE on offspring NDBD risk.

Methods: Using multivariable logistic regression analysis of survey data from the National Longitudinal Study of Adolescent to Adult Health - a longitudinal study of a nationally representative sample of adolescents in grades 7-12 in the United States, we examined the association between indicators of paternal
ACE and offspring NDBD. Our study included an analysis of 3,130 children born to 1,865 males.

Results: Children born to men with histories of childhood emotional or physical abuse exhibited the highest adjusted odds for NDBD, $1.4(1.0,2.1)$ and $1.7(1.2,2.6)$, respectively. The association between paternal childhood emotional or physical abuse, and offspring NDBD remained significant for male but not female offspring when the analysis was stratified by gender.

Conclusions: Paternal early life stress demonstrated a significant sex-specific association with offspring NDBD. Future work is necessary to understand the underlying mechanisms through which these childhood experiences negatively impact offspring health.

\section{P-47: Pregnant Women's Perspectives on Paternal Involvement in Prenatal Care: Implications for Policy and Practice}

Tova B. Walsh (presenting author), ${ }^{1}$ Emma Carpenter, ${ }^{2}$ Molly Costanzo, ${ }^{2}$ and Lanikque Howard ${ }^{2}$

${ }^{1}$ School of Social Work, Rutgers University; and ${ }^{2}$ School of Social Work, University of Wisconsin-Madison

Background and Objectives: Supportive paternal prenatal involvement can positively impact maternal and child outcomes. Paternal participation in prenatal care is increasing, yet little research exists to understand the mothers' experiences and preferences. We examine pregnant women's perspectives on paternal involvement in prenatal care, and identify policy and practice implications.

Methods: We interviewed 22 pregnant women after a routine prenatal exam approximately midway through pregnancy. Interviews addressed when and how fathers participated in prenatal care, how mothers experienced paternal involvement, perceptions of the medical providers' treatment of fathers, and preferences for paternal involvement. Grounded theory guided data analysis was conducted using NVivo.

Results: Nearly all participants reported some paternal involvement in prenatal care. Participants perceived this involvement as both support for the mother and an assuming role of the father. Most participants valued paternal involvement as an opportunity for fathers to learn how to support a healthy pregnancy, bond with their developing baby, and share joy and worries with the mothers. Participants generally felt that fathers were made to feel welcome, and wished for providers to be inclusive of fathers. An important exception is one participant's belief that her abusive partner's involvement would be harmful.

Conclusions: Results suggest the importance for prenatal care that is supportive of partner involvement when appropriate, and centered on the needs and preferences of pregnant women. Prenatal care presents an opportunity to reach men to enhance their positive involvement in pregnancy, support for mothers, and preparation of parenthood. Prenatal care also provides an opportunity to screen for abuse and referrals for services.

\section{P-48: Associations Between Medication Class and Uncontrolled Blood Pressure Among Older Men and Women Treated for Established Hypertension Using Single Class Antihypertensive Medications}

LaKeisha Williams (presenting author), ${ }^{1,2}$ Erin Peacock, ${ }^{2}$ Lydia Bazzano, ${ }^{3}$ Daniel Sarpong, ${ }^{1}$ Marie Krousel-Wood ${ }^{2,3,4}$ 
${ }^{1}$ Division of Clinical and Administrative Sciences, College of Pharmacy, Xavier University of Louisiana; ${ }^{2}$ Department of Medicine, School of Medicine, ${ }^{3}$ Department of Epidemiology, School of Public Health and Tropical Medicine, Tulane University; and ${ }^{4}$ Research Division, Ochsner Health System

Background and Objectives: Antihypertensive medications are effective in lowering blood pressure (BP), yet some medication classes may be more effective than others in older women versus older men. We examined associations between medication class and uncontrolled BP in men and women taking single class antihypertensive medications from the Cohort Study of Medication Adherence Among Older Adults.

Methods: A cross-sectional analysis of participants $(n=375)$ taking angiotensin-converting enzyme inhibitors (ACEI), beta blockers (BB), calcium channel blockers (CCB), diuretics, or angiotensin receptor blockers (ARB) was conducted. Uncontrolled $\mathrm{BP}$ was defined as systolic $\mathrm{BP} \geq 140 \mathrm{mmHg}$ or diastolic $\mathrm{BP} \geq 90 \mathrm{mmHg}$. Overall and sex-stratified multivariable logistic regression models adjusted for sociodemographic, clinical, and medication adherence variables assessed associations between medication class and uncontrolled BP.

Results: Participants were 59.0\% female, 26.3\% black, and mean age of 74.5 years. Prevalence of uncontrolled BP was 32.5\% overall: $35.1 \%$ for women, $28.6 \%$ for men. Antihypertensive medication use varied by class (overall, women, men): ACEI $(31.1 \%, 27.5 \%, 36.4 \%)$, diuretics $(21.8 \%, 27.0 \%$, $14.3 \%)$, СCB $(21.3 \%, 21.2 \%, 21.4 \%), \mathrm{BB}(16.8 \%, 14.0 \%$, $20.8 \%)$, and ARB $(9.0 \%, 10.4 \%, 7.1 \%)$. In separate models and compared to diuretics, there were significantly higher odds of uncontrolled BP for overall participants taking BB (OR 2.93, CI $1.32,6.51$ ), CCB (OR 4.22, CI 2.04, 8.74), ARB (OR 2.55, CI $1.00,6.50$ ), or ACEI (OR 2.01, CI 0.99, 4.08); results were qualitatively similar in women and men.

Conclusions: Compared to diuretics, use of other antihypertensive medication classes was associated with uncontrolled BP among older men and women with hypertension.

\section{P-49: Gender Differences in Access and Outcomes of High-Risk Surgical Oncology Procedures}

Richelle T. Williams (presenting author), ${ }^{1}$ Joseph Levy, ${ }^{2}$ Nader Hanna, ${ }^{1}$ Brajesh Lal, ${ }^{1}$ and C. Daniel Mullins ${ }^{2}$

${ }^{1}$ Department of Surgery, University of Maryland Medical Center; and ${ }^{2}$ Pharmaceutical Health Services Research Department, University of Maryland School of Pharmacy

Background and Objectives: Although studies have demonstrated an inverse relationship between surgical volume and outcomes, gender differences for high-risk surgical oncology procedures have not been explored within the volume-outcome paradigm. This study characterizes gender differences in access/ utilization for two high-risk surgical oncology procedures and assesses the impact of gender on short-term outcomes (length of stay [LOS], cost, mortality, and complications).

Methods: We analyzed patients undergoing pancreatectomy or esophagectomy using the New York State Inpatient Database, 2009-2014. Gender differences in access/utilization were examined, comparing sociodemographic and hospital characteristics.
Generalized linear models were used to assess the independent effect of gender on short-term outcomes.

Results: There were 8,281 pancreatectomies $(4,062$ men, 4,219 women) and 1,948 esophagectomies (1,528 men, 420 women). Women undergoing pancreatectomy were more likely to be black ( $12 \%$ vs. $9 \%$ ), have a lower comorbidity index (3.6 vs. 4.1$)$, and have an elective hospital admission ( $85 \%$ vs. $81 \%)$. In contrast, women undergoing esophagectomy more likely had an emergency (10\% vs. $6 \%)$ or urgent (3\% vs. $1 \%)$ admission. After risk adjustment, women had shorter LOS ( -1.3 days, $P<0.001)$, lower cost $(-\$ 5034, P<0.001)$, lower mortality (OR $0.67, P<0.001$ ), and fewer complications (OR 0.76, $P<0.001$ ) following pancreatectomy. LOS, cost, and mortality were not significantly different for men and women undergoing esophagectomy, but women had fewer complications (OR 0.82, $P<0.01)$. There was no gender difference in utilization of highvolume hospitals for either procedure.

Conclusions: A greater proportion of women had urgent or emergency admissions for esophagectomy. Women had similar or better short-term outcomes for both procedures.

\section{P-50: Race and Sex Differences in Maternal Mediated Childhood Obesity (MMCO)}

ClarLynda Williams-DeVane (presenting author), ${ }^{1}$ Michele Josey, ${ }^{2}$ and Cathrine Hoyo ${ }^{3}$

${ }^{1}$ Biological and Biomedical Sciences Department and

${ }^{2}$ Biomedical Biotechnology Research Institute, North Carolina Central University; and ${ }^{3}$ Center for Human Health, North Carolina State University

Background and Objectives: Childhood obesity is an epidemic in the United States creating a medical burden of more than $\$ 14$ billion a year as of 2010. It is an epidemic that disproportionately affects minorities and those of lower socioeconomic status. Although diet and exercise interventions have had limited success, the etiology and complexity of childhood obesity and its disparities are not well understood. To address childhood obesity, the Newborn Epigenetic Study (NESt) was initiated to prospectively test the influence of in utero environmental exposures on DNA methylation profiles in newborns. However, analyzing DNA methylation data requires the correct classification of obesity status in the child and the associated risk based on maternal Body Mass Index (BMI). We hypothesized that the relationship between childhood obesity status and maternal BMI classification is both sex and race specific.

Methods: We calculated the race and sex-specific odds ratios of risk for children in NESt becoming overweight or obese by age 4. Furthermore, we identified ideal cutoffs for maternal BMI utilizing Receiver Operating Characteristic curves.

Results: The odds ratios of obesity risk between Caucasian and African American, as well as male and female children, are significantly different. Furthermore, the optimal maternal BMI cutoff for the prediction of children becoming overweight or obese by age 4 is significantly different in Caucasian and African American mothers.

Conclusions: This work will lead to a better understanding of the maternal influences of childhood obesity and more efficient analysis of DNA methylation data. 